\title{
Self-consistent Green's function embedding for advanced electronic structure methods based on a dynamical mean-field concept
}

\author{
Wael Chibani, ${ }^{1, *}$ Xinguo Ren,,${ }^{1,2}$ Matthias Scheffler, ${ }^{1}$ and Patrick Rinke ${ }^{1,3}$ \\ ${ }^{1}$ Fritz-Haber-Institut der Max-Planck-Gesellschaft, Faradayweg 4-6, D-14195 Berlin, Germany \\ ${ }^{2}$ Key Laboratory of Quantum Information, University of Science and Technology of China, Hefei 230026, China \\ ${ }^{3}$ COMP/Department of Applied Physics, Aalto University, P.O. Box 11100, Aalto FI-00076, Finland
}

(Received 2 June 2015; revised manuscript received 22 March 2016; published 6 April 2016)

\begin{abstract}
We present an embedding scheme for periodic systems that facilitates the treatment of the physically important part (here a unit cell or a supercell) with advanced electronic structure methods, that are computationally too expensive for periodic systems. The rest of the periodic system is treated with computationally less demanding approaches, e.g., Kohn-Sham density-functional theory, in a self-consistent manner. Our scheme is based on the concept of dynamical mean-field theory formulated in terms of Green's functions. Our real-space dynamical mean-field embedding scheme features two nested Dyson equations, one for the embedded cluster and another for the periodic surrounding. The total energy is computed from the resulting Green's functions. The performance of our scheme is demonstrated by treating the embedded region with hybrid functionals and many-body perturbation theory in the $G W$ approach for simple bulk systems. The total energy and the density of states converge rapidly with respect to the computational parameters and approach their bulk limit with increasing cluster (i.e., computational supercell) size.
\end{abstract}

DOI: 10.1103/PhysRevB.93.165106

\section{INTRODUCTION}

Density-functional theory (DFT) has become a widely applied electronic structure theory method due to the balance between accuracy and computational efficiency of local and semilocal approximations such as the local-density (LDA) and generalized gradient approximations (GGA). However, LDA and GGAs suffer from certain intrinsic limitations such as the self-interaction error [1-3], the absence of the derivative discontinuity in the exchange-correlation potential [4-6], the lack of long-range van der Waals interactions [7-9], and the absence of image effects [10-12]. These shortcomings limit the predictive power of LDA and GGAs, in particular for localized electrons as found in $d$ - or $f$-electron systems [13-17] or for adsorbates and surfaces [18-21]. Furthermore, DFT is inherently a ground-state method and therefore of limited applicability for excited states and spectra. More advanced electronic structure methods that overcome one or several of the mentioned shortcomings exist, but they are typically computationally much more demanding and thus limited to small systems sizes or a subset of electronic degrees of freedom. To overcome the efficiency-accuracy conundrum, much effort has been devoted to combine the best of both worlds, that is, to merge local and semilocal DFT approximations (DFA) with advanced electronic structure methods [22-35]. The latter includes, e.g., quantum chemistry methods [36], advanced exchange-correlation functionals of the fourth and fifth rung Jacob's ladder [37], and Green's function based many-body perturbation theory [38].

We here advocate the concept of embedding. In this divide and conquer approach, the full system is divided into two parts: a small embedded region, which is treated with advanced, computationally demanding approaches, and an embedding environment that is treated with computationally more efficient

\footnotetext{
*chibani@fhi-berlin.mpg.de
}

approaches. A schematic illustration of the embedding concept is shown in Fig. 1.

Following this general principle, various embedding schemes have been developed in the past [22-35,39]. They differ in scope (i.e., area of application), on how they treat the coupling between the embedded region and the surrounding, and in the approaches used to describe the two regions. In (bio)chemistry, for example, one of the most popular embedding schemes combines quantum mechanics (QM) and classical molecular mechanics (MM). The embedded region is treated quantum mechanically and the surrounding by MM [23-27]. In surface science, fully quantum mechanical schemes are more prevalent, e.g., for the description of surface adsorbates. They divide space into regions for advanced and less advanced electronic structure approaches and differ mostly on how these two regions are coupled, e.g., through maximal exchange overlap [28], density embedding [29], or cluster extrapolation [30,31]. In solid-state physics, dynamical mean-field theory [32-34] offers a natural embedding framework by mapping an infinite, correlated lattice model into an impurity model [40] immersed into a self-consistently determined mean-field bath. When DFA is chosen as the mean field, dynamical mean-field theory (DMFT) becomes material specific [34,41,42]. The embedding is achieved by means of Green's functions facilitating the calculation of spectra, band structures, but also phase diagrams. Recently, Zgid and Chan [22] proposed to use DMFT as an embedding framework for quantum-chemistry approaches such as the configuration-interaction (CI) method. They since proposed a simplified DMFT scheme based on density-matrix embedding to access static properties (e.g., the ground-state energy and its derivatives) [35]. However, at present all DMFT approaches use a downfolding procedure to a low-energy subspace, which is treated on the model Hamiltonian level.

We here extend the DMFT concept to couple two firstprinciples regions. Our idea is similar to that of Zgid and Chan [22], but we explore the possibility of using DMFT 


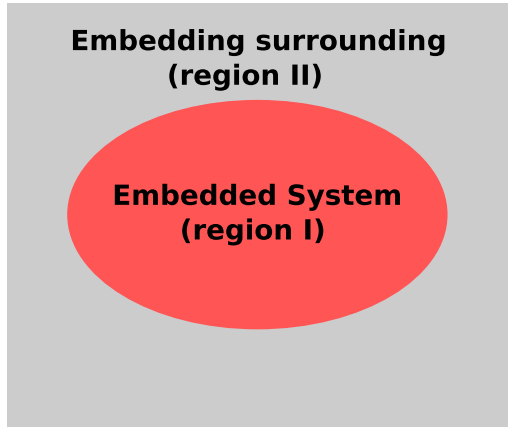

FIG. 1. Schematic depiction of the embedding concept. The embedded region (red) is treated with a more accurate theory, whereas the surrounding (gray) is calculated with less accurate and thus computationally less expensive theories. The key challenge of our and all previous embedding approaches is the appropriate treatment of the red/gray boundary.

as a general embedding scheme for advanced first-principles electronic structure methods. These can be advanced DFT exchange-correlation functionals or excited-state methods based on the $G W$ approach [38], which are still computationally too expensive for large-scale systems. The difference to previous DMFT schemes is that we treat the unit cell as the local, embedded region, that is coupled to the rest of the periodic system via the DMFT framework. All electrons in the embedded region are treated at the same quantum mechanical level, which removes the necessity of defining a downfolded subspace and does not require any doublecounting corrections. This is possible in our scheme because we do not attempt to solve the local many-body problem exactly, which is a standard practice in conventional DMFT schemes. The pros and cons of our strategy will be further discussed in Sec. II A.

We here present the concept of our real-space dynamical mean-field embedding (RDMFE) approach and its implementation in the all-electron Fritz Haber Institute $a b$ initio molecular simulations (FHI-AIMS) code [43-45]. We first benchmark it for hybrid density functionals for which we have a periodic reference [46]. Then, we apply our scheme to the $G W$ approach. Due to its intrinsic self-consistency, our RDMFE approach yields a self-consistent $G W$ solution, which is a much coveted approach for solids right now. While fully self-consistent $G W$ implementations for molecules are slowly emerging [47-52], we are only aware of one recent implementation of fully self-consistent $G W$ for solids [53,54], which is, however, limited to small unit cells due to its computational expense. An alternative, approximate way to achieve self-consistency within $G W$ is the so-called quasiparticle self-consistent $G W(\mathrm{QPsc} G W)$ scheme [55,56], which was recently been widely applied to solids. We here present self-consistent $G W$ spectra and ground-state energies for simple solids.

As is well known, the DMFT approach works best for the Hubbard-type model Hamiltonian where only local Coulomb interactions are present. One may question its ultimate usefulness to be employed as an embedding scheme for first-principles Hamiltonians. We think the answer to this question is positive for the following reasons. First, what we wish to capture within RDMFE is not the full hybrid or $G W$ self-energies, but rather their difference to the LDA or GGA exchange-correlation (XC) potentials. This difference is relatively short ranged and easier to capture. Second, our formulation is similar to cluster DMFT, whereby all "interatomic" correlations within the embedded cluster are included, and we explicitly check the convergence of the calculated results with respect to the cluster size. Third, we consider this formulation as a natural extension of our previous work (Refs. [30,31]) where corrections to LDA/GGAs brought by more advanced methods were only carried out for bare clusters. Fourth, the RDMFE formalism can be easily extended to embed a more sophisticated beyond- $G W$ treatment into a $G W$ environment. Such a $G W$-based RDMFE scheme would then treat long-range screening on the $G W$ level and short-ranged beyond- $G W$ correlations in the local region.

The rest of the paper is organized as follows: The detailed formalism of our Green's function based embedding scheme is derived in Sec II. Section III presents the computational details. Benchmark results, including both total energies and band structures for simple bulk systems, are discussed in Sec. IV. In Sec. V, we contrast our approach with the aforementioned embedding schemes. Section VI concludes the paper.

\section{SELF-CONSISTENT GREEN'S FUNCTION EMBEDDING IN REAL SPACE}

\section{A. General concept}

In its original formulation [34], DMFT is a Green's function method for correlated model Hamiltonians that uses the locality of the electronic interaction to embed a local onsite region of the Hubbard lattice, typically a single $d$ or $f$ level, into a periodic electronic bath defining a self-consistent scheme. Treating the onsite region locally facilitates the use of computationally very demanding and at the same time very accurate (essentially exact) methods such as continuous time quantum Monte Carlo [57], direct diagonalization, or renormalization group techniques [58]. The localized region is then coupled through a hybridization self-energy to the surrounding electronic bath, which is treated with computationally more efficient methods. In the last few years, DMFT has proven to be very successful in describing the spectral properties of solids with localized $d$ and $f$ states [59-62].

Further developments include the extension of the DMFT formulation from a single site to clusters $[63,64]$, the combination of DMFT with first-principles approaches [65-67], and the formulation of DMFT for nanostructures [68]. A key feature of all flavors of DMFT is that the local problem (a single site or a cluster) is solved essentially exactly. To this end, it is necessary to further reduce the electronic degree of freedom of the local problem to a correlated subspace. This is actually a highly nontrivial issue, and a recent discussion along this line can be found in Ref. [69].

On the other hand, if one takes a step back, and does not require that the local problem be solved exactly, then the projection to a correlated subspace is not any more necessary. The price to pay is that the thus formulated approach may not be able to treat "strongly correlated problems" such as the Mott-Hubbard metal-insulator transition. However, one 


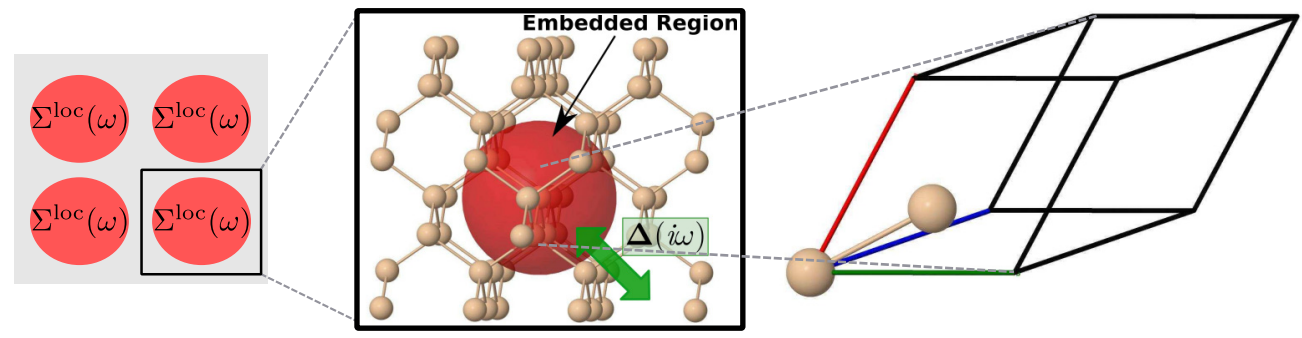

FIG. 2. The DMFT embedding concept for a Si unit cell. The atoms in the unit cell (red region) constitute the embedded submanifold. Each unit cell of the periodic system is treated as a localized region, i.e., only local interactions $\Sigma^{\text {loc }}$ are treated. The unit cells are coupled to the rest of the system via the hybridization self-energy $\Delta(i \omega)$ (green arrow).

should note that there are a large number of problems where "Mott physics" is not dominating, yet approaches beyond LDA/GGAs are required to get quantitatively accurate results. These approaches include quantum chemistry methods [36], advanced exchange-correlation functionals of the fourth and fifth rung Jacob's ladder [37], and Green's function based many-body perturbation theory [38]. They all treat electronic systems at $a b$ initio level, but are restricted to small systems. In this work, we make use of the concept of DMFT (more precisely cluster DMFT) and formulate it as a Green's function embedding scheme for the above-noted $a b$ initio approaches. The motivation is to extend the reach of these approaches to unprecedented system size. In this work, we take hybrid functionals and the $G W$ approximation as examples. We consider the unit cell (or any computational supercell that can span the whole space when periodically repeated) as embedded cluster (see Fig. 2). For clusters bigger than the primitive unit cell, our formalism is similar to cluster DMFT (for a review, see Ref. [64]), in particular the cellular DMFT scheme [63]. Consequently, the translational symmetry of the original lattice is lost and only that of the supercell is retained (for further discussions, see Sec. IV A). The difference of our approach to cluster DMFT is that we do not attempt to solve the quantum mechanical problem of the cluster exactly, and hence are able to treat all electronic degrees of freedom on equal footings.

Compared to the traditional embedding approaches discussed in the Introduction, popular in computational chemistry and materials science, our approach permits the charge flow from one region to the other and therefore naturally incorporates the boundary between the two regions. No special treatment is necessary for atoms on the boundary, nor is it a problem with the boundary cuts covalent bonds.

\section{B. Embedding scheme based on DMFT}

\section{Green's function in a nonorthogonal basis set}

The embedding framework of DMFT is most conveniently formulated in terms of Green's functions. In a finite (and generally nonorthogonal) basis set $\left\{\phi_{i}\right\}$, the Green's function $G\left(\mathbf{r}, \mathbf{r}^{\prime}, i \omega\right)$ can be expanded as

$$
G\left(\mathbf{r}, \mathbf{r}^{\prime}, i \omega\right)=\sum_{i, j} \phi_{i}(\mathbf{r}) G_{i j}(i \omega) \phi_{j}\left(\mathbf{r}^{\prime}\right),
$$

where $G_{i j}(i \omega)$ is the matrix form of the Green's function. Here, we use the Green's function on the imaginary frequency axis for computational convenience and without loss of generality.
For a noninteracting Hamiltonian $H_{i j}^{0}=\left\langle\phi_{i}\left|\hat{H}^{0}\right| \phi_{j}\right\rangle$ [e.g., the Kohn-Sham (KS) Hamiltonian], the corresponding noninteracting Green's function $G^{0}$ in its matrix form satisfies

$$
\sum_{k}\left[(i \omega+\mu) S_{i k}-H_{i k}^{0}\right] G_{k j}^{0}=\delta_{i j}
$$

where $S_{i j}=\left\langle\phi_{i} \mid \phi_{j}\right\rangle$ is the overlap matrix of the basis functions, and $\mu$ is the chemical potential. Using the Dyson equation that connects the noninteracting Green's function $G^{0}$ with the fully interacting one $(G)$, we obtain

$$
\sum_{k}\left[(i \omega+\mu) S_{i k}-H_{i k}^{0}-\Sigma_{i k}(i \omega)\right] G_{k j}(i \omega)=\delta_{i j},
$$

where $\Sigma(i \omega)$ is the electronic self-energy. For periodic systems, the Hamiltonian and the Green's functions are characterized by a Bloch $\mathbf{k}$ vector in the first Brillouin zone of reciprocal space. Equation (3) thus becomes

$\sum_{k}\left[(i \omega+\mu) S_{i k}(\mathbf{k})-H_{i k}^{0}(\mathbf{k})-\Sigma_{i k}(\mathbf{k}, i \omega)\right] G_{k j}^{\mathrm{lat}}(\mathbf{k}, i \omega)=\delta_{i j}$,

with the lattice Green's function $G^{\text {lat }}(\mathbf{k}, i \omega)$. Our implementation is based on the all-electron FHI-AIMS code package [43], which uses numerical atom-centered orbitals (NAOs) as its basic functions. The basis functions $\left\{\phi_{i}\right\}$ will thus be NAOs in our work.

\section{2. “Onsite" Green's function for a periodic system}

The k-dependent Green's function and self-energy in Eq. (4) can be Fourier transformed to real space,

$$
\begin{aligned}
G_{i j}^{\mathrm{lat}}\left(\mathbf{R}_{i}-\mathbf{R}_{j}, i \omega\right) & =\frac{1}{N_{1 . \mathrm{BZ}}} \sum_{\mathbf{k}}^{1 . \mathrm{BZ}} e^{i\left(\mathbf{R}_{i}-\mathbf{R}_{j}\right) \cdot \mathbf{k}} G_{i j}^{\mathrm{lat}}(\mathbf{k}, i \omega), \\
\Sigma_{i j}\left(\mathbf{R}_{i}-\mathbf{R}_{j}, i \omega\right) & =\frac{1}{N_{1 . \mathrm{BZ}}} \sum_{\mathbf{k}}^{1 . \mathrm{BZ}} e^{i\left(\mathbf{R}_{i}-\mathbf{R}_{j}\right) \cdot \mathbf{k}} \Sigma_{i j}(\mathbf{k}, i \omega),
\end{aligned}
$$

where $\mathbf{R}_{i}$ and $\mathbf{R}_{j}$ are Bravais lattice vectors denoting the unit cells in which the basis functions $i$ and $j$ are located. Please note that the Bravais vectors here do not necessarily correspond to primitive unit cells. When choosing a supercell as the embedded region, $\mathbf{R}_{i}$ and $\mathbf{R}_{j}$ then refer to the superlattice formed by the repetition of the supercells. $N_{1 \mathrm{BZ}}$ is the number of $\mathbf{k}$ points in the first Brillouin zone (1.BZ). The concept of DMFT is based on the fact that the lattice self-energy becomes 
local, or $\mathbf{k}$ independent, in infinite dimension $(D=\infty)$ [32]. For a crystal with translational symmetry this implies

$$
\Sigma_{i j}\left(\mathbf{R}_{i}-\mathbf{R}_{j}, i \omega\right)=\Sigma_{i j}^{\text {loc }}(i \omega) \delta_{\mathbf{R}_{i}, \mathbf{R}_{j}} .
$$

Thus, the self-energy is nonzero only if the two basis functions originate from the same unit cell. We call this the local (loc) or "onsite" self-energy, following the terminology of the model Hamiltonian studies. But, here $i$ and $j$ do not necessarily sit on the same atom, nor within the same primitive unit cell, if a supercell cluster is used. In this limit, the whole periodic system can be mapped onto an effective impurity model of a local unit cell dynamically coupled to an effective "external" potential arising from the rest of the crystal.

The first step to establish this mapping is to define the "onsite" Green's function, i.e., $G_{i j}\left(\mathbf{R}_{i}-\mathbf{R}_{j}, i \omega\right)$ with $\mathbf{R}_{i}=$ $\mathbf{R}_{j}$. Using the locality of the self-energy and Eq. (4), we obtain the following expression for the onsite Green's function:

$$
\begin{aligned}
& G_{i j}^{\mathrm{on}-\mathrm{site}}(i \omega) \\
& =\frac{1}{N_{1 . \mathrm{BZ}}} \sum_{\mathbf{k}}^{1 . \mathrm{BZ}} G_{i j}^{\mathrm{lat}}(\mathbf{k}, i \omega) \\
& =\frac{1}{N_{1 . \mathrm{BZ}}} \sum_{\mathbf{k}}^{1 . \mathrm{BZ}}\left[(i \omega+\mu) S(\mathbf{k})-H^{0}(\mathbf{k})-\Sigma^{\mathrm{loc}}(i \omega)\right]^{-1}
\end{aligned}
$$

In the DMFT context, this equation is also known as the $\mathbf{k}$ integrated Dyson equation. So far we have not specified $H^{0}$. In our embedding scheme, the environment is treated by KS-DFA in the LDA or the Perdew-Burke-Ernzerhof (PBE) [70] GGA. A natural choice of $H^{0}$ is thus the KS Hamiltonian $H^{\mathrm{KS}}(\mathbf{k})$ within LDA or GGA, that contains the kinetic-energy operator, the external potential $\left(v_{\mathrm{ext}}\right)$, the Hartree potential $\left(v_{\mathrm{H}}\right)$, and the $\mathrm{XC}$ potential $\left(v_{\mathrm{XC}}\right)$ :

$$
H^{\mathrm{KS}}(\mathbf{k})=-\frac{1}{2} \nabla^{2}+v_{\mathrm{ext}}(\mathbf{k})+v_{\mathrm{H}}(\mathbf{k})+v_{\mathrm{XC}}(\mathbf{k}) .
$$

Next, we need to define $\Sigma^{\text {loc }}(i \omega)$ in Eq. (7). If we start from $H^{\mathrm{KS}}(\mathbf{k})$, the onsite self-energy becomes the difference between the dynamic, complex many-body exchange-correlation selfenergy $\Sigma_{\mathrm{XC}}(\mathbf{k}, i \omega)$ and the $\mathrm{KS} \mathrm{XC}$ potential, i.e.,

$$
\begin{aligned}
\Sigma^{\mathrm{loc}}(i \omega) & =\frac{1}{N_{1 . \mathrm{BZ}}} \sum_{\mathbf{k}}^{1 . \mathrm{BZ}}\left[\Sigma_{\mathrm{XC}}(\mathbf{k}, i \omega)-v_{\mathrm{XC}}^{\mathrm{KS}}(\mathbf{k})\right] \\
& =\Sigma_{\mathrm{XC}}^{\mathrm{loc}}(i \omega)-v_{\mathrm{XC}}^{\mathrm{loc}} .
\end{aligned}
$$

Using Eqs. (7) and (9), we finally obtain

$$
\begin{aligned}
G_{i j}^{\text {onsite }}(i \omega)= & \frac{1}{N_{1 . \mathrm{BZ}}} \sum_{\mathbf{k}}^{1 . \mathrm{BZ}}\left[(i \omega+\mu) S(\mathbf{k})-H^{\mathrm{KS}}(\mathbf{k})\right. \\
& \left.-\Sigma_{\mathrm{XC}}^{\mathrm{loc}}(i \omega)+v_{\mathrm{XC}}^{\mathrm{loc}}\right]^{-1} .
\end{aligned}
$$

At this point, we would like to briefly comment on the doublecounting issue, which arises commonly when combining DMFT with first-principles DFT. The problem occurs because the contribution of the $\mathrm{KS} \mathrm{XC}$ potential within the correlated model space, where the self-energy obtained from the DMFT calculation is defined, is not clear. However, from the above derivation, one can see that neither the usage of the KS Green function as the reference, nor the local approximation of the self-energy correction, will necessarily lead to double counting, provided that the KS XC potential and the manybody self-energy can be defined and computed in the same electronic space. This is the case of the RDMFE scheme, where both the local $\mathrm{KS} \mathrm{XC}$ potential and the hybrid/ $G W$ self-energies are defined for the embedded cluster. Thus, we conclude that our scheme is free of double-counting ambiguities.

\section{Embedded Green's function}

In the DMFT formalism, a periodic system is viewed as a periodically repeated cluster (here the unit or supercell) dynamically embedded into a self-consistently determined environment. The coupling between the embedded subsystem and its surrounding environment is described by a so-called bath Green's function $\mathcal{G}(i \omega)$, connecting the Green's function of the embedded cluster $G^{\mathrm{emb}}(i \omega)$ and the local self-energy via

$$
[\mathcal{G}(i \omega)]^{-1}=\left[G^{\mathrm{emb}}(i \omega)\right]^{-1}+\Sigma^{\mathrm{loc}}(i \omega) .
$$

Here, the local self-energy $\Sigma^{\text {loc }}(i \omega)$ is the same as introduced in Eq. (9). The self-consistency condition of DMFT requires that the Green's function of the embedded cluster $G^{\mathrm{emb}}(i \omega)$ equals the onsite Green's function as given in Eq. (7):

$$
G^{\mathrm{emb}}(i \omega)=G^{\mathrm{on}-\text { site }}(i \omega) .
$$

Alternatively, one can also use a so-called hybridization function $\Delta(i \omega)$ to describe the coupling between the embedded cluster and its environment, which provides a more intuitive picture. $\Delta(i \omega)$ is closely related to the bath Green's function $\mathcal{G}(i \omega)$ :

$$
[\mathcal{G}(i \omega)]^{-1}=(i \omega+\mu) S-H_{0}^{\text {cluster }}-\Delta(i \omega) .
$$

In Eq. (13), $H_{0}^{\text {cluster }}$ is the Hamiltonian of the bare cluster describing the noninteracting unit cell, i.e., without the $v_{\mathrm{XC}}^{\mathrm{KS}}$ contribution and without the presence of the other atoms from neighboring unit cells (see Fig. 2). This corresponds to the onsite term of the Hamiltonian of the periodic system, and in practice can be conveniently obtained from the $\mathbf{k}$-dependent Hamiltonian

$$
H_{0}^{\text {cluster }}=\frac{1}{N_{1 . \mathrm{BZ}}} \sum_{\mathbf{k}}^{1 . \mathrm{BZ}}\left[H^{\mathrm{KS}}(\mathbf{k})-v_{\mathrm{XC}}^{\mathrm{KS}}(\mathbf{k})\right] .
$$

Using Eqs. (11)-(13), we obtain the following expression for the Green's function of the embedded cluster:

$$
\begin{aligned}
{\left[G^{\mathrm{emb}}(i \omega)\right]_{i j}^{-1}=} & {\left[(i \omega+\mu) S-H_{0}^{\text {cluster }}\right.} \\
& \left.-\Sigma^{\mathrm{loc}}\left[G^{\mathrm{emb}}\right](i \omega)-\Delta(i \omega)\right]_{i j} .
\end{aligned}
$$

Here, we have explicitly indicated that the local self-energy is a functional of the embedded Green's function. Thus, Eq. (15) has to be solved self-consistently, which corresponds to the inner loop of Fig. 3. The functional dependence of $\Sigma^{\text {loc }}(i \omega)$ on $G^{\mathrm{emb}}(i \omega)$ is given by the actual approximation for the localized region, which will be the topic of the next section. However, already here we see that our RDMFE approach lends itself to those advanced electronic structure methods that can be expressed by (self-consistent) Green's functions. 


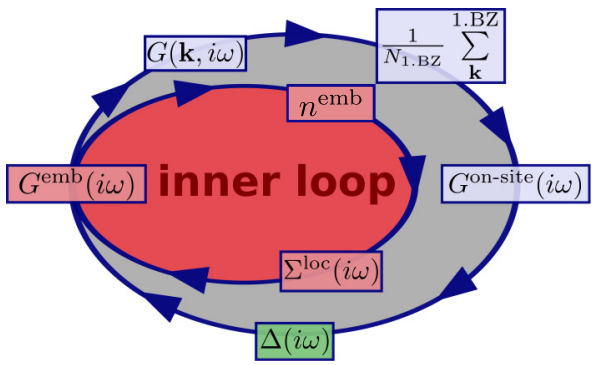

FIG. 3. The embedded [Eq. (15)] and the onsite [Eq. (7)] Green's functions define two Dyson equations that form two nested loops. The two loops are iterated until self-consistency is reached.

Another point we would like to emphasize is the choice of the cluster overlap matrix $S$ in Eq. (13). We found when updating the chemical potential of the cluster in the inner loop that we needed to define the cluster overlap matrix as $S=\left[\frac{1}{N_{1} \mathrm{BZ}} \sum_{\mathbf{k}}^{1 . \mathrm{BZ}} S^{-1}(\mathbf{k})\right]^{-1}\left[\right.$ and not simply $\left.S=\frac{1}{N_{1} \mathrm{BZ}} \sum_{\mathbf{k}}^{1 . \mathrm{BZ}} S(\mathbf{k})\right]$ as done by Kotliar et al. [41] to enforce the correct asymptotic behavior of $\Delta(i \omega)$, i.e., $\lim _{\omega \rightarrow \infty} \Delta(\omega) \rightarrow 0$.

\section{Local self-energy}

So far we had not specified the approximation for the local self-energy in Eq. (15). In our scheme the choice for $\Sigma^{\text {loc }}\left[G^{\text {emb }}\right]$ can be quite flexible. In other words, we could use any approximation that goes beyond LDA and GGAs. However, our framework lends itself to Green's function based approaches. This includes density-matrix and density-based approaches because both quantities can easily be extracted from the Green's function. Below we report on two different examples, namely, hybrid density functionals that mix a fraction of exact exchange with GGA semilocal exchange [70-72] and the $G W$ approximation [38]. In practice, we could also go beyond $G W$, e.g., by including the screened secondorder exchange (SOSEX) self-energy that was developed recently [73].

We here use the PBE hybrid functional family (PBEh) [74], whose most prominent functional is PBE0 [75]. We will also use the short-ranged range-separated hybrid functional family by Heyd, Scuseria, and Ernzerhof (HSE) [72]. In PBEh, the local self-energy in Eq. (9) is given by

$$
\begin{aligned}
\Sigma_{\mathrm{PBEh}}^{\mathrm{loc}}(\alpha) & =\left[\alpha \Sigma_{\mathrm{X}}^{\mathrm{loc}}+(1-\alpha) v_{\mathrm{X}}^{\mathrm{loc}}+v_{\mathrm{C}}^{\mathrm{loc}}\right]-v_{\mathrm{XC}}^{\mathrm{loc}} \\
& =\alpha\left(\Sigma_{\mathrm{X}}^{\mathrm{loc}}-v_{\mathrm{X}}^{\mathrm{loc}}\right) .
\end{aligned}
$$

In Eq. (16), $v_{\mathrm{X}}^{\text {loc }}$ is the onsite part of the GGA exchange, and $\Sigma_{\mathrm{X}}^{\text {loc }}$ is the exact-exchange matrix given by

$$
\left[\Sigma_{\mathrm{X}}^{\mathrm{loc}}\right]_{i j}=\sum_{k, l}\langle i k \mid l j\rangle n_{k l}^{\mathrm{emb}},
$$

where $\langle i k \mid l j\rangle$ are two-electron four-orbital integrals, and $n_{k l}^{\mathrm{emb}}$ is the density matrix of the embedded cluster which can be obtained from the embedded Green's function

$$
n_{i j}^{\mathrm{emb}}=-\frac{i}{2 \pi} \int d \omega G^{\mathrm{emb}}(i \omega)_{i j} e^{i \omega \tau^{+}} .
$$

The two-electron Coulomb repulsion integrals are evaluated using the resolution of identity (RI) technique in FHI-AIMS as documented in Ref. [45]. The PBE0 functional is obtained for $\alpha=0.25$ [75].

The extension to an HSE type self-energy is straightforward. In HSE, a range-separation parameter is introduced that cuts off the exact-exchange contribution at long distances. The range is controlled via the screening parameter $\gamma$ so that the local exchange self-energy becomes

$$
\Sigma_{\mathrm{X}}^{\mathrm{loc}}(\gamma)=\Sigma_{\mathrm{X}}^{\mathrm{loc}, \mathrm{SR}}(\gamma)+\Sigma_{\mathrm{X}}^{\mathrm{loc}, \mathrm{LR}}(\gamma),
$$

with SR and LR denoting the short- and long-range parts, respectively. If we now replace $\Sigma_{\mathrm{X}}^{\text {loc,LR }}(\gamma)$ by $v_{\mathrm{X}}^{\text {loc,LR }}$ and introduce the $\alpha$ parameter again, the local HSE self-energy assumes the following form:

$$
\Sigma_{\mathrm{HSE}}^{\mathrm{loc}}(\alpha, \gamma)=\alpha\left(\Sigma_{\mathrm{X}}^{\mathrm{SR}, \mathrm{loc}}(\gamma)-v_{\mathrm{X}}^{\mathrm{SR}, \mathrm{loc}}(\gamma)\right) .
$$

Furthermore, we employ the $G W$ approximation for the local self-energy. Here, the computation of the $G W$ self-energy for a given input embedded Green's function follows the self-consistent $G W$ implementation for finite systems in FHIAIMS [45,50]. On the imaginary-time axis, the $G W$ self-energy for the embedded cluster is obtained as

$$
\left[\Sigma_{\mathrm{XC}}^{\mathrm{loc}}(i \tau)\right]_{i j}=\frac{i}{2 \pi} \sum_{l k \mu \nu} M_{i k}^{\mu} M_{l j}^{v} G_{k l}^{\mathrm{emb}}(i \tau)\left[W^{\mathrm{loc}}(i \tau)\right]_{\mu \nu} .
$$

Here, $\mu, v$ indices refer to the auxiliary basis set used to expand the screened Coulomb interaction $W^{\text {loc }}$ in the RI approach [45,50]. Furthermore, $M_{i k}^{\mu}$ are the three-index coefficients obtained as

$$
M_{i k}^{\mu}=\sum_{v}(i k \mid \mu) V_{\mu \nu}^{-1 / 2},
$$

where

$$
(i k \mid \mu)=\int d \mathbf{r} d \mathbf{r}^{\prime} \frac{\phi_{i}(\mathbf{r}) \phi_{k}(\mathbf{r}) P_{\mu}\left(\mathbf{r}^{\prime}\right)}{\left|\mathbf{r}-\mathbf{r}^{\prime}\right|}
$$

and

$$
V_{\mu \nu}=\int d \mathbf{r} d \mathbf{r}^{\prime} \frac{P_{\mu}(\mathbf{r}) P_{\nu}\left(\mathbf{r}^{\prime}\right)}{\left|\mathbf{r}-\mathbf{r}^{\prime}\right|}
$$

with $\left\{P_{\mu}(\mathbf{r})\right\}$ being the auxiliary basis functions. For $W$ we thus obtain

$$
W_{\mu \nu}^{\mathrm{loc}}(i \omega)=\sum_{\alpha} V_{\mu \alpha}\left[1-\Pi^{\mathrm{loc}}(i \omega)\right]_{\alpha \nu}^{-1},
$$

where $\Pi^{\text {loc }}(i \omega)$ the irreducible polarizability, whose Fourier transform in the time domain is directly determined by the embedded Green's function

$$
\Pi_{\mu \nu}^{\mathrm{loc}}(i \tau)=-i \sum_{i j l m} M_{i l}^{\mu} M_{j m}^{\nu} G_{i j}^{\mathrm{emb}}(i \tau) G_{l m}^{\mathrm{emb}}(-i \tau)
$$

\section{Self-consistency loops}

In our formalism, Eqs. (15) and (17) or (21) define an additional inner self-consistency loop for the local self-energy as depicted in Fig. 3. Good convergence is achieved by a linear mixing

$$
\Sigma_{n+1}^{\mathrm{loc}}=\lambda \Sigma_{n}^{\mathrm{loc}}+(1-\lambda) \Sigma_{n-1}^{\mathrm{loc}},
$$

with a mixing parameter $\lambda=0.5$. More advanced mixing schemes could be implemented as well, but we found that 


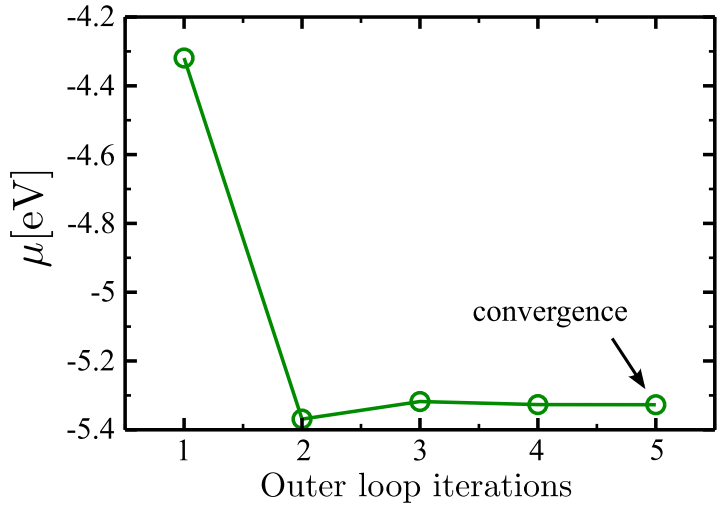

FIG. 4. Typical change in the chemical potential during the selfconsistency cycle for a bulk Si calculation. Convergence is reached after five iterations of the outer loop. Already at the second iteration the chemical potential is close to its converged value.

linear mixing works well for the examples presented in this work. When the inner loop reaches convergence, we feed the resulting $\Sigma^{\text {loc }}$ back into the onsite GF and iterate the main loop further using the same mixing as for the inner loop.

Finally, it is worth mentioning that the onsite Green's function as defined in Eq. (7) requires that our $\Sigma^{\text {loc }}$ in the onsite Green's function in the zeroth iteration should be $\Sigma_{0}^{\text {loc }}=V_{\text {XC }}^{\text {loc }}$. Figure 3 shows a sketch of the embedding scheme as described above. During the self-consistency cycle, we compute the particle number $N_{\mu}$, a quantity that is obtained from the embedded Green's function via

$$
N_{\mu}=-\frac{i}{2 \pi} \operatorname{Tr} \int d \omega G_{i j}^{\mathrm{emb}}(\omega, \mu) e^{-i \omega 0^{+}} .
$$

To ensure particle-number conservation, we need to update the electron chemical potential every time we receive a converged self-energy from the inner loop. For the present test cases, we found that the change in the chemical potential is relatively small, as demonstrated in Fig. 4 for bulk silicon (Si). However, we expect it to be more important for metallic systems.

\section{E. Total-energy calculation}

Once self-consistency in the embedding scheme is reached, we can compute the total energy of the entire systems (the embedded cluster plus the environment) using the converged lattice Green's function $G_{i j}(\mathbf{k}, \omega)$. The actual total-energy expression depends on the chosen methodology used in the embedded region. For hybrid density functionals, we have

$$
E_{\mathrm{tot}}^{\mathrm{hyb}}=\frac{1}{N_{1 . \mathrm{BZ}}}\left[\sum_{\mathbf{k}} \sum_{i, j} t_{j i}(\mathbf{k}) n_{i j}(\mathbf{k})\right]+E_{\mathrm{es}}[n]+E_{\mathrm{XC}}^{\mathrm{hyb}}\left[n_{i j}\right],
$$

where

$$
t_{j i}(\mathbf{k})=\sum_{\mathbf{R}}\left\langle\phi_{j}(\mathbf{r})\left|-\frac{\nabla^{2}}{2}\right| \phi_{i}(\mathbf{r}-\mathbf{R})\right\rangle e^{i \mathbf{k} \cdot \mathbf{R}}
$$

is the matrix form of the kinetic energy operator, $E_{\mathrm{es}}$ the electrostatic (Hartree plus external) energy, and $E_{\mathrm{XC}}^{\text {hyb }}$ the XC energy. In Eq. (29), $n_{i j}$ is the k-dependent global density matrix

$$
n_{i j}(\mathbf{k})=\int \frac{d \omega}{2 \pi} G_{i j}^{\mathrm{lat}}(\mathbf{k}, i \omega) e^{-i \omega 0^{+}},
$$

and $n$ is the electron density obtained from $n_{i j}(\mathbf{k})$. We note that Eq. (29) is the exact total-energy expression for the hybrid density functional, and the only approximation is that the density matrix $n_{i j}(\mathbf{k})$ (and hence electron density $n$ ) is obtained from the RDMFE scheme and not from a periodic hybrid functional calculation.

However, Eq. (29) cannot be directly applied since evaluating $E_{\mathrm{XC}}^{\text {hyb }}$ as a functional of the $\mathbf{k}$-dependent density matrix $n_{i j}(\mathbf{k})$ requires the computation of the exact-exchange energy for the entire periodic system, which is exactly what we are trying to avoid here. Instead of evaluating $E_{\mathrm{XC}}^{\text {hyb }}\left[n_{i j}(\mathbf{k})\right]$ in full, we thus only compute the change of $E_{\mathrm{XC}}^{\text {hyb }}$ with respect to the local or semilocal (LDA or GGA) energy in the embedded region. This is the main approximation of our approach, which is consistent with the spirit of the local self-energy correction in the RDMFE scheme, and is suggested by the nearsightedness of the XC energy of a bulk system (although the exact-exchange energy is probably not the most nearsighted self-energy we could have chosen) [76].

The Hartree energy depends on the electron density in a highly nonlocal way and it is questionable if a local treatment can be applied to the Hartree energy at all. Therefore, for simplicity, we omit possible changes in the Hartree and the external energy for now, assuming that the electron density given by the local or semilocal approximation is already sufficient.

Finally, we are left with the kinetic energy term which also changes when moving from local or semilocal to hybrid functionals. For consistency, kinetic and XC energy should be taken together. In our scheme, we evaluate the changes of the kinetic and XC energy caused by the local self-energy correction within the embedded region.

Based on the above considerations, we propose the following approximate total-energy expression for embedded hybrid functional calculations:

$$
\begin{gathered}
E_{\mathrm{tot}}^{\mathrm{hyb}} \approx E_{\mathrm{tot}}^{\mathrm{KS}}+\left[\sum_{i j} t_{j i}\left(n_{i j}^{\mathrm{emb}}-n_{i j}^{\mathrm{KS}}\right)\right] \\
+E_{\mathrm{XC}}^{\mathrm{hyb}}\left[n_{i j}^{\mathrm{emb}}\right]-E_{\mathrm{XC}}^{\mathrm{KS}}\left[n^{\mathrm{KS}}\right],
\end{gathered}
$$

where $n_{i j}^{\mathrm{emb}}$ is the embedded density matrix as defined in Eq. (18), and $n_{i j}^{\mathrm{KS}}$ is the onsite density matrix of KS-LDA/GGA calculations. $n^{\mathrm{KS}}(\mathbf{r})$ is obtained from the onsite KS density matrix

$$
n^{\mathrm{KS}}(\mathbf{r})=\sum_{i j} \phi_{i}(\mathbf{r}) n_{i j}^{\mathrm{KS}} \phi_{j}(\mathbf{r}),
$$

and $E_{\mathrm{XC}}^{\mathrm{KS}}\left[n^{\mathrm{KS}}\right]$ is thus restricted to the embedded region.

For $G W$ we can proceed in an analogous fashion

$$
\begin{aligned}
E_{\mathrm{tot}}^{G W}= & \frac{1}{N_{1 . \mathrm{BZ}}}\left[\sum_{\mathbf{k}} \sum_{i, j}\left[t_{j i}(\mathbf{k}) n_{i j}(\mathbf{k})\right]\right] \\
& +E_{\mathrm{es}}[n]+E_{\mathrm{XC}}^{G W}\left[G_{i j}^{\mathrm{lat}}\right],
\end{aligned}
$$


where

$$
E_{\mathrm{XC}}^{G W}\left[G_{i j}^{\mathrm{lat}}\right]=\frac{1}{2} \sum_{\mathbf{k}} \frac{1}{N_{1 . \mathrm{BZ}}} \int \frac{d \omega}{2 \pi} \Sigma_{j i}(\mathbf{k}, \omega) G_{i j}(\mathbf{k}, \omega) e^{-i \omega 0^{+}}
$$

following directly from the Galitskii-Migdal (GM) formula [77]. Similar to the hybrid functional case, we will not take the full $\mathbf{k}$ dependence in $E_{\mathrm{XC}}^{G W}\left[G_{i j}^{\mathrm{lat}}(\mathbf{k}, i \omega)\right]$ into account. Instead, we adopt the same philosophy as before and make a local approximation

$$
\begin{aligned}
E_{\mathrm{tot}}^{G W} \approx & E_{\mathrm{tot}}^{\mathrm{KS}}+\sum_{i j} t_{j i}\left(n_{i j}^{\mathrm{emb}}-n_{i j}^{\mathrm{KS}}\right) \\
& +E_{\mathrm{XC}}^{G W}\left[G_{i j}^{\mathrm{emb}}(i \omega)\right]-E_{\mathrm{XC}}^{\mathrm{KS}}\left[n^{\mathrm{KS}}\right],
\end{aligned}
$$

where

$$
E_{\mathrm{XC}}^{G W}\left[G_{i j}^{\mathrm{emb}}\right]=\frac{1}{2} \int \frac{d \omega}{2 \pi i} \Sigma_{j i}^{\mathrm{loc}}(i \omega) G_{i j}^{\mathrm{emb}}(i \omega) e^{-i \omega 0^{+}} .
$$

To summarize this part, in RDMFE the total energy of the entire system can in principle be obtained from the lattice Green's function. However, in practice, approximations are needed to make the problem tractable. The expressions for hybrid functional and $G W$ calculations proposed above are consistent with the local nature of the self-energy approximation in RDMFE, but their performance needs to be checked in practical calculations. Future work needs to revisit total-energy calculations in RDMFE.

\section{COMPUTATIONAL DETAILS}

We used tight FHI-AIMS integration grids for all our RDMFE calculations. For the embedded PBEh and HSE self-energy we used the tier 1 basis set. Figure 5 shows the embedded PBEh total and cohesive energies with increasing basis set size. The lattice Green's functions were represented on a logarithmic frequency grid with 40 points. The total-energy calculations for 2- and 8-atom silicon unit cells and the density of states (DOS) calculations for 8- and 16-atom unit cells were performed on a $4 \times 4 \times 4$ k-point grid, which we increased to $10 \times 10 \times 10$ for DOS calculations in the 2 -atom unit cell. $G W$ calculations were performed in a tier 3 basis set with 40 frequency/time points in the inner loop and the same number of $\mathbf{k}$ points as for PBEh. The linear mixing parameter $\lambda$ was fixed to 0.5 , which gave reasonably fast convergence. The periodic PBE and PBE0 reference calculations were performed using the tier 1 basis set and a $12 \times 12 \times 12 \mathbf{k}$ mesh.

Densities of states are obtained in two different ways. In PBEh and HSE we obtain a self-energy that defines a converged k-dependent embedded Hamiltonian via the converged lattice Green's function once the self-consistency cycle is converged. For the PBEh self-energy we can directly diagonalize the embedded Hamiltonian $H^{\text {embed }}(\mathbf{k})=H^{0}(\mathbf{k})+$ $\Sigma_{\mathrm{PBEh}}^{\text {loc }}$ at each k point, which yields k-dependent eigenvalues and eigenstates. The resulting density of states (DOS) is $n[\epsilon(\mathbf{k})]=\sum_{\nu} \delta\left[\epsilon(\mathbf{k})-\epsilon_{\nu}(\mathbf{k})\right]$, where $v$ labels the eigenstates of $H^{\text {embed }}(\mathbf{k})$. To make the comparison to experiment easier,
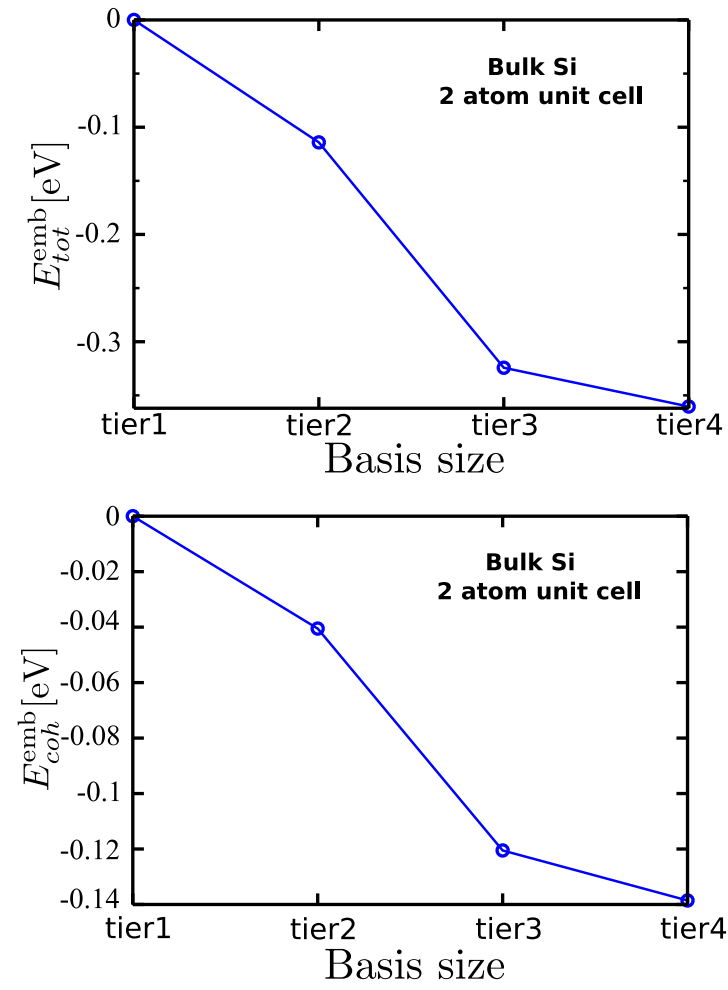

FIG. 5. RDMFE(PBE0) total energy (upper panel) and cohesive energy (lower panel) for bulk $\mathrm{Si}$ with a two-atom unit cell as function of the basis size. The energy zero is set at the value of the tier 1 basis.

we introduce a Gaussian broadening

$$
g_{v}(\tilde{\epsilon})=\int d \mathbf{k} \exp \left(-\frac{\tilde{\epsilon}-\epsilon_{\nu}(\mathbf{k})}{\sqrt{2} \sigma}\right)^{2}
$$

to obtain the $\operatorname{DOS} N(\tilde{\epsilon})=\sum_{v} g_{\nu}(\tilde{\epsilon})$. In this work we use a Gaussian broadening of $\sigma=0.2 \mathrm{eV}$.

For the $G W$ self-energy, the spectrum at each $\mathbf{k}$ point is directly given by the Green's function as

$$
A(\mathbf{k}, \omega)=-\frac{1}{\pi} \operatorname{Tr}\left\{\operatorname{Im} G^{\text {lat }}(\mathbf{k}, \omega)\right\} .
$$

To determine $G^{\text {lat }}(\mathbf{k}, \omega)$ on the real-frequency axis, we analytically continue the self-energy from the imaginary to the real axis. In practice, we fit a two-pole model, that has proven to work very well for the systems we tested, to each matrix element of the self-energy $[45,78]$

$$
\Sigma(i \omega) \approx \sum_{n}^{2} \frac{\alpha_{n}}{i \omega-\beta_{n}},
$$

where $\alpha_{n}$ and $\beta_{n}$ are complex fitting parameters. We then evaluate Eq. (40) for real frequencies and solve Dyson's equation for $G^{\text {lat }}(\mathbf{k}, \omega)$. The spectral function subsequently follows from a $\mathbf{k}$ summation $A(\omega)=\sum_{\mathbf{k}} A(\mathbf{k}, \omega)$, which we convolute with Gaussians as

$$
\tilde{A}(\tilde{\omega})=\int d \omega e^{-\left(\frac{\tilde{\omega}-\omega}{\sqrt{2} \sigma}\right)^{2}} A(\omega),
$$

with a broadening that we choose to be $\sigma=0.01 \mathrm{eV}$ to obtain a DOS $\tilde{A}(\tilde{\omega})$ that we can compare with experiment. 


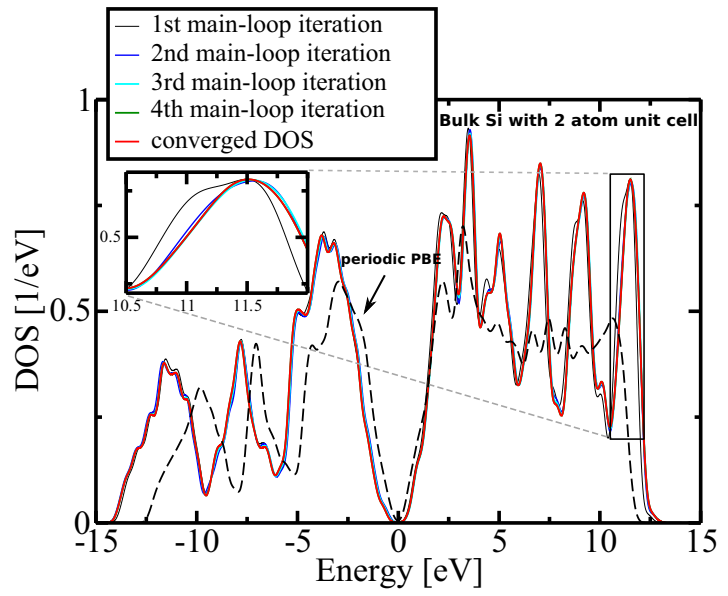

FIG. 6. RDMFE(PBE0) DOS comparison at each iteration of the main loop. Convergence was achieved after five main-loop iterations.

\section{RESULTS}

Having introduced the concept of RDMFE and our implementation in the previous sections, we now turn to benchmark calculations for hybrid functionals, for which we have an independent, periodic reference in FHI-AIMS [46]. Then, we present self-consistent $G W$ calculations for which such a periodic reference does not yet exist in FHI-AIMS. We choose bulk $\mathrm{Si}$ as test system since it is a reliable and well-studied reference case.

\section{A. Density of states and band structures}

We begin our benchmark tests by calculating the DOS at each iteration to investigate its evolution with each embedding cycle. Figure 6 shows the DOS at different iterations of the outer loop for a two-atom unit cell of silicon. We observe that the largest change occurs at the first iteration when moving from PBE to our embedded PBE0 DOS. For subsequent iterations, the DOS changes are much smaller.

When comparing the converged RDMFE(PBE0) DOS for the two-atom unit cell with the periodic PBE and PBE0 DOS shown in Fig. 7, we observe that the band width and band gap are larger than in $\mathrm{PBE}$ and are closer to the PBE0 reference.

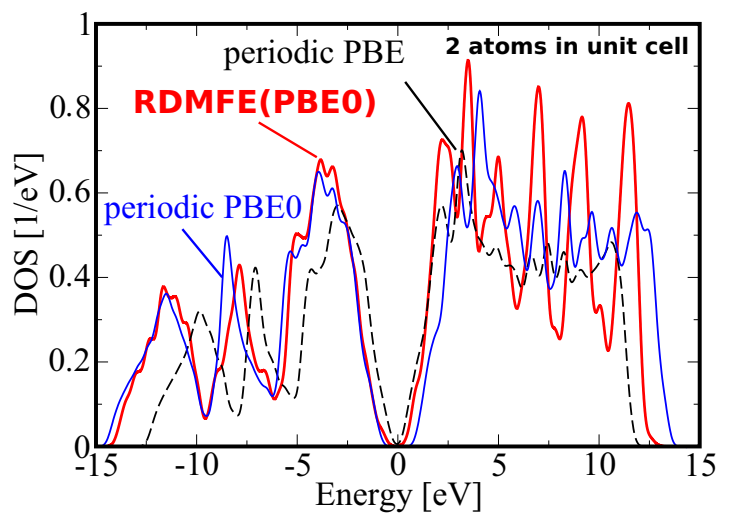

FIG. 7. Comparison of the RDMFE(PBE0) DOS, the periodic $\mathrm{PBE}$, and the periodic PBE0 DOS for a two-atom unit cell.
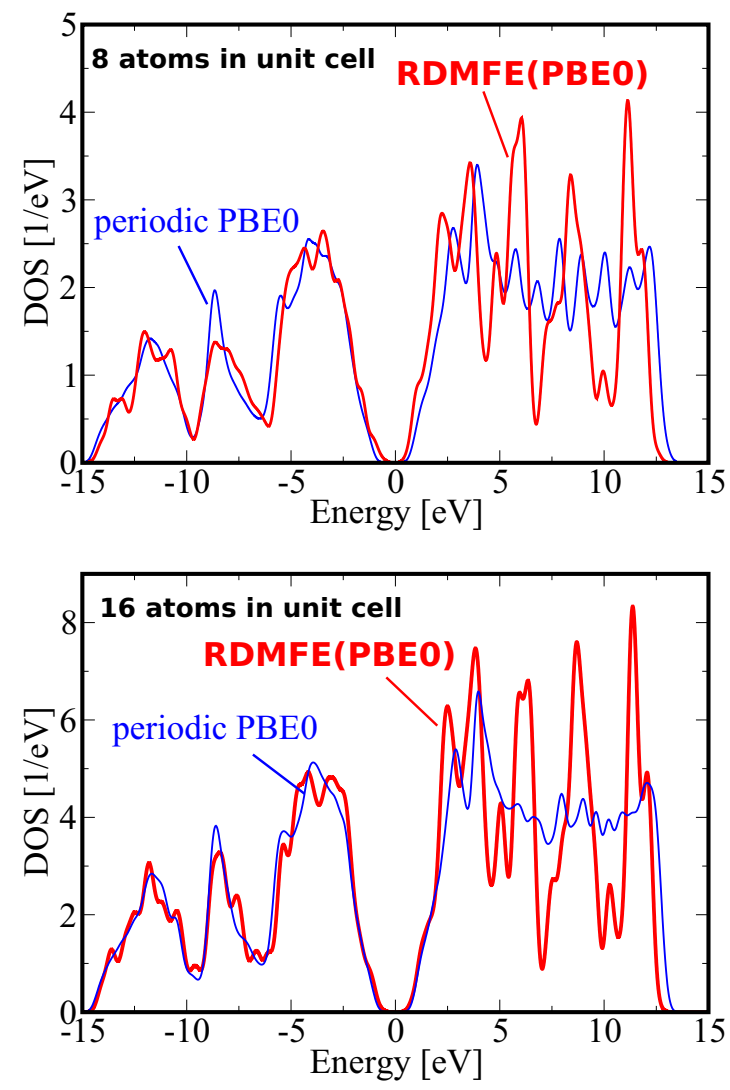

FIG. 8. Comparison of the RDMFE(PBE0) DOS, with the periodic PBE0 DOS for an 8-atom unit cell (upper panel) and a 16-atom unit cell (lower panel).

When increasing the unit-cell size to 8 and 16 atoms (see Fig. 8) the difference between the embedded DOS and the periodic PBE0 DOS reduces systematically. The resulting RDMFE band gaps for different unit cell sizes are compared with the PBE and the PBE0 values in Table I. With increasing unit-cell size, the band gap increases and approaches the PBE0 value.

Next, we consider the band structure for the two-atom unit cell shown in the upper panel of Fig. 9. We see the same trend as for the DOS: the band gap and the band width approach PBE0 and so do the bands in general. However, at some highsymmetry points the degeneracy of certain bands is lifted. The origin of this degeneracy lifting is the break of the crystal symmetry that we introduce with the local self-energy. It is a well-known artifact and has been discussed extensively in the context of cellular and cluster DMFT [80,81]. The local self-energy simply does not "know" about the symmetry of the crystal and can therefore not enforce it. The solution to the problem is then obvious: the approximation of the locality of the self-energy needs to be improved. If the self-energy would extend over a larger region (i.e., supercell) it would acquire more information about the crystal symmetry. Then, the degeneracy splitting should reduce. In the two lower panels of Fig. 9, we present an unfolded band structure [82] for the 16- and 32-atom unit cells. We indeed observe a reduction in the splitting for both the 16- and 32-atom unit cells. However, while the degeneracy is fully restored for some high-symmetry points, it is still broken for others such as the $X$ and $Z$ points. 
TABLE I. Comparison between PBE, PBE0, and RDMFE(PBE0) for different unit-cell sizes for the indirect band gap of silicon. The experimental value [79] is shown for reference.

\begin{tabular}{lcccccr}
\hline \hline & & & \multicolumn{3}{c}{ RDMFE(PBE0) } & Experiment (at 300 K) [79] \\
\cline { 3 - 5 } & PBE & PBE0 & 2 atoms & 8 atoms & 16 atoms & 1.12 \\
\hline \hline Band gap (eV) & 0.68 & 1.85 & 1.20 & 1.257 & 1.569 & 12 \\
\hline \hline
\end{tabular}

To obtain the $\operatorname{RDMFE}(G W)$ band structure, we calculate the $\operatorname{RDMFE}(G W)$ spectral function at each $\mathbf{k}$ point. We then turn this information into a two-dimensional (2D) plot by color coding the intensity. The result is illustrated in Fig. 10 for bulk $\mathrm{Na}$ (with one atom in the unit cell) along the $\Gamma-N$ path and shows that the two-pole fit [Eq. (40)] for the analytic continuation is also applicable to metals. Our $\operatorname{RDMFE}(G W)$ band structure exhibits a larger band width compared to periodic PBE calculation and to previous quasiparticle selfconsistent $G W$ calculations [55,83]. However, we like to reiterate that our results are preliminary in the sense that the calculations have been performed for only one atom in the unit cell and the unit-cell size convergence will have to be checked carefully.

We will now turn to the $G W$ spectra. The total spectral function for bulk $\mathrm{Si}$ with two atoms in the unit cell at the first iteration is shown in Fig. 11. Since Dyson's equation has been solved once, this spectrum is not equivalent to perturbative $G_{0} W_{0}$ spectra and we would expect to see plasmon satellites. The spectrum shows a broad peak between -40 and $-30 \mathrm{eV}$, which has been identified as plasmon satellite [84,85]. Such satellites are completely absent in KS band structures or in $G_{0} W_{0}$ because $G_{0} W_{0}$ only corrects the KS states and does not yield new states. The energy range of the RDMFE satellite agrees well with previous periodic $G W$ calculations [84,85] and demonstrates that our dynamic, local RDMFE framework can capture nonlocal phenomena such as plasmon satellites. For sc $G W$ the converged DOS is also shown in Fig. 11. As demonstrated by Holm and von Barth [86] for the electron gas, full self-consistency in $G$ and $W$ leads to a deterioration in the $G W$ spectral function due to the neglect of vertex corrections. Thus, the fact that the plasmon satellite disappears at selfconsistency is not surprising. We obtain a band gap of $\sim 0.9 \mathrm{eV}$ for the two-atom unit cell, which is close to the experimental value of $\sim 1.12 \mathrm{eV}$ [87]. This comparison together with the one between the indirect band gap from our calculation and experiment [79] are presented in Table II.

\section{B. Embedded total energies:}

For $\operatorname{sc} G W$ we currently do not have a periodic reference to compare to, as alluded to before. We can, however, construct another test case and benchmark against our sc $G W$ implementation for finite systems $[49,50]$, where the total energy was computed from the Galitskii-Migdal formula [77]. We achieve this by considering the molecular limit of a unit cell, i.e., the limit of an isolated unit cell with a lattice constant of $\sim 20 \AA$. The benchmark results for $\mathrm{He}, \mathrm{H}_{2}$, and $\mathrm{Na}_{2}$ are presented in Table III, which shows the XC components that enter the total energy as given by Eq. (37). $\Sigma_{\mathrm{XC}}^{\mathrm{sc} G W}$ is the molecular $\mathrm{sc} G W$ XC self-energy and $G^{\mathrm{sc} G W}(i \omega)$ the corresponding Green's function at convergence. $\Sigma_{\mathrm{XC}}^{\text {loc }}$ is local XC self-energy and $G^{\mathrm{emb}}(i \omega)$ the embedded Green's function both obtained at convergence of the RDMFE cycle. Table III illustrates that the components entering the embedded total energy agree almost to the meV level with the corresponding components from the finite systems $\mathrm{sc} G W$ calculation, which demonstrates the reliability and robustness of our implementation.

We then investigated the convergence of the total energy with respect to the increase of the unit-cell size for RDMFE PBE0 and scGW. For embedded PBE0, we performed calculations for bulk Si up to 32 atoms in the unit cell, whereas for $G W$ we considered bulk He in the fcc structure up to 64-atom unit cells. To reach larger systems, a full parallelization of our implementation would be required. The upper panel of Fig. 12 shows the comparison of our embedded PBE0 cohesive energy with the periodic PBE and the periodic PBE0 energy. We also include a third reference in which we added the kinetic and $\mathrm{XC}$ energy of a PBE0 calculation to the PBE energy, which most closely resembles our RDMFE approximation. We see that with increasing unit-cell size the embedded cohesive energy approaches the periodic PBE0 value, but then dips below. This is not surprising since our embedded cohesive energy does not account for changes in the electrostatic energy. Instead, the RDMFE curve approaches the PBE0 reference value from which the electrostatic change has been removed. However, the convergence to the periodic limit is relatively slow. This can be related to the long-range nature of the HF exact exchange as we will show later on using a range-separated self-energy (see the discussion of Fig. 13). For the $G W$ self-energy, however, the total energy seems to converge much faster and only changes in a very small range. This is shown in the lower panel of Fig. 12.

To better visualize the interplay between locality and unit-cell size in our scheme we also present results of the HSE range-separated exact-exchange self-energy. We vary the range-separation parameter to model different degrees of locality, but keep the percentage $\alpha$ of exact-exchanged fixed. We then translate the range-separation parameter into a radius $R_{\text {sphere }}$ in real-space using the relation $\gamma=R_{\text {sphere }}^{-1}$ and determine the number of atoms that fit inside. We have considered range-separation parameters $\gamma$ that correspond to spheres enclosing 2-, 4-, 8-, and 16-atom unit cells. For each $\gamma$, the resulting embedded total energy is plotted in Fig. 13 as a function of the size of the unit cell (upper panel). The lower panel shows the volume of the surrounding sphere for the different $\gamma$ parameters and for the different unit-cell sizes. We indeed observe that the total energy converges faster with unit-cell size, the shorter the range of the nonlocality in the HSE self-energy. This proves that RDMFE becomes a viable option for self-energies, whose range only encompasses a few 


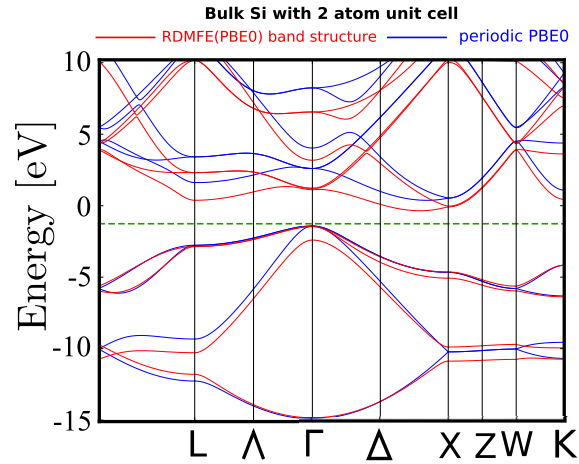

Bulk Si with 16 atom unit cell

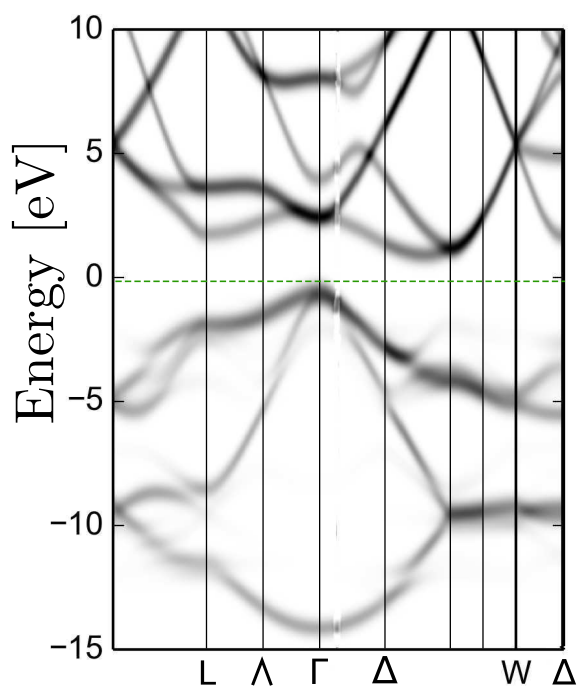

Bulk Si with 32 atom unit cell

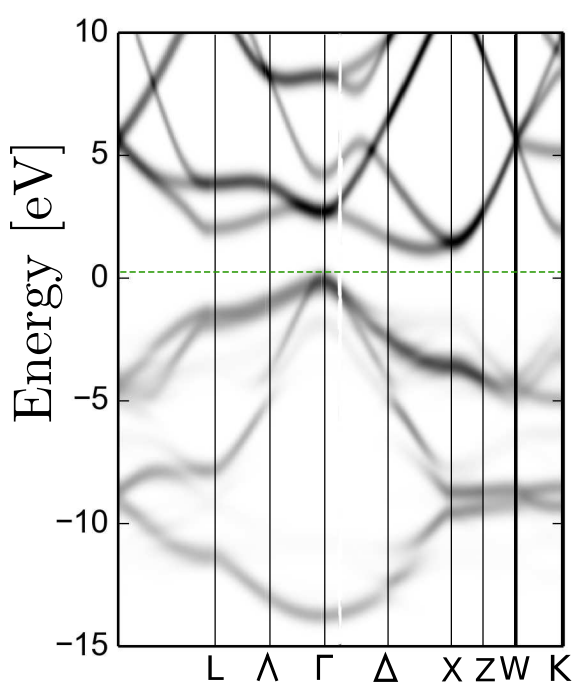

FIG. 9. Upper panel: the RDMFE(PBE0) band structure for bulk Si compared to the periodic PBE0 one. The local self-energy breaks the translation symmetry and the degeneracy gets shifted at some high-symmetry $\mathbf{k}$ points. Middle panel: the RDMFE(PBE0) unfolded band structure for the 16-atom unit cell. The degeneracy shifting gets reduced compared to the 2-atom case. Lower panel: the RDMFE(PBE0) unfolded band structure for the 32-atom unit cell. Also, here the degeneracy is restored in most of the high-symmetry points.

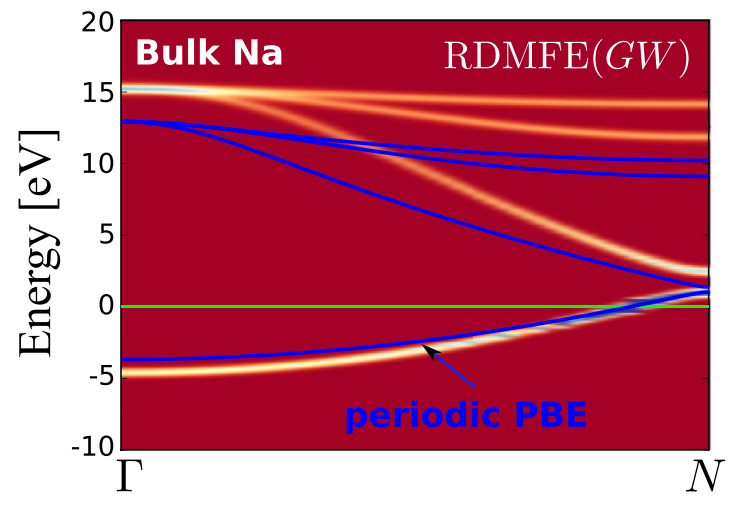

FIG. 10. The band structure of bulk Na determined from the k-resolved $\operatorname{RDMFE}(G W)$ spectral function along the $\Gamma-N$ direction, compared to the periodic PBE one (blue solid line). Both band structures are aligned at the same chemical potential.

nearest atoms. In that sense, PBE0 had been the toughest test because its range is infinite.

Finally, we briefly discuss cohesive properties as described by the RDMFE scheme. In Fig. 14, we show the total energy of bulk $\mathrm{Si}$ as a function of the lattice constant for RDMFE(PBE0), $\operatorname{sc} G W$, and for periodic PBE0. For RDMFE(PBE0) (upper panel) our calculations for a 2-atom unit cell already give a minimum that is below the PBE one and very close to the experimental value of $5.43 \AA$ [88] (see Table IV). However, for an 8- and a 16-atom unit cell, the lattice constant reduces slightly and reaches its converged value already for the 8 -atom unit cell. For $\operatorname{RDMFE}(G W)$, the minimum for the 2-atom unit cell (lower panel) also lies below the PBE value and already agrees fortuitously well with the experimental value. Our $\operatorname{RDMFE}(G W)$ lattice constant is slightly larger than that reported by a recent periodic self-consistent $G W$ calculation [53]. We also performed a Birch-Murnaghan [89] fit of the total-energy curves to extract the bulk moduli for $\operatorname{RDMFE}(\mathrm{PBE} 0)$ and $\operatorname{RDMFE}(G W)$. The resulting values are reported in Table IV. For the RDMFE(PBE0) calculation, the bulk modulus of the 2-atom unit cell is smaller than the

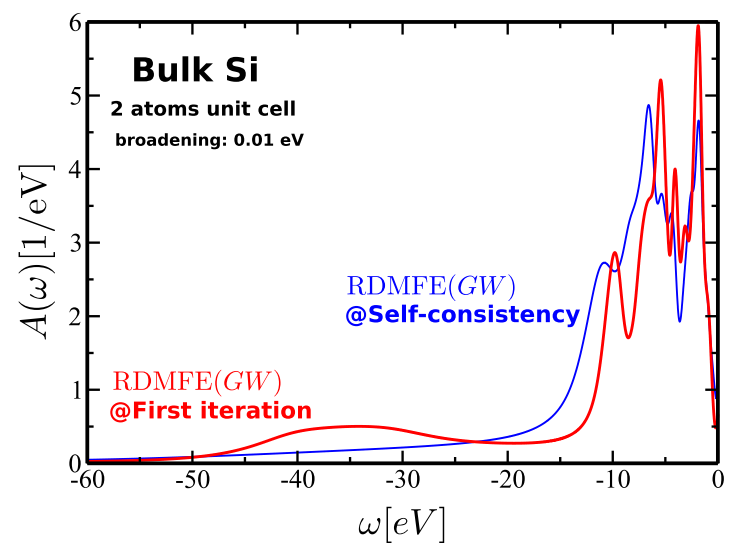

FIG. 11. Gaussian broadened (with broadening $\sigma=0.01 \mathrm{eV}$ ) quasiparticle spectrum for the $\operatorname{RDMFE}(G W)$ self-energy at first iteration (red curve) and at self-consistency (blue curve). Only occupied states are shown. 
TABLE II. Direct and indirect band gaps as calculated from RDMFE for the $G W$ self-energy. Comparison is made with the periodic sc $G W$ work of Kutepov et al. [53] and the quasiparticle self-consistent $G W$ calculation of Kotani et al. [56] and experiment.

\begin{tabular}{lcccc}
\hline \hline Band gap & $\operatorname{RDMFE}(G W)$ & Periodic sc $G W[53]$ & $Q P \operatorname{sc} G W[56]$ & Experiment $($ at 300 K) \\
\hline Direct $\left(\Gamma_{15 c}\right)(\mathrm{eV})$ & 3.70 & & 3.47 & $3.40[79]$ \\
Indirect $\left(E_{g}\right)(\mathrm{eV})$ & 0.90 & 1.55 & 1.25 & $1.12[87]$ \\
\hline \hline
\end{tabular}

experimental value. For the 8-atom unit cell, however, the bulk modulus is considerably increased before it takes a value that is reasonably close to experiment for the 16-atom unit cell. For all unit-cell sizes the RDMFE(PBE0) bulk modulus is larger than the periodic PBE0 value. Conversely, the $\operatorname{RDMFE}(G W)$ bulk modulus for the 2-atom unit cell is considerably smaller that the periodic $\operatorname{sc} G W$ reference and the experimental value. This suggests that an investigation of larger unit cells is mandatory and will be carried out in future work.

\section{DISCUSSION}

We have presented an embedding scheme for periodic systems that builds on DMFT. In our approach, the electron interacting across periodically repeated unit cells is mapped onto an onsite problem, in which the electrons only interact directly in one unit cell, but are dynamically coupled to a periodic bath of electrons. The coupling between the embedded system and the surrounding is constructed naturally by means of Green's functions. Due to its dynamic nature, the bath can exchange electrons with the embedded region. Our embedding scheme is most suitable for systems with periodic boundary conditions, as the translational symmetry with respect to the computational supercell is preserved. Furthermore, we transfer the nonlocality of a self-energy into a frequency dependence, a concept that has previously been explored by Gatti et al. [91] and in the spectral density-functional theory of Kotliar et al. [92]. We note that the only approximation introduced in our scheme is that the nonlocal XC coupling between neighboring unit cells (or computational supercells) is included only at the KS GGA level, and neglected in the more advanced (here hybrid functional or $G W$ ) treatment. In other words, the self-energy correction to the GGA XC potential is $\mathbf{k}$ independent, an intrinsic feature of DMFT.

We now compare our scheme to other embedding schemes. For the hybrid QM:MM approach a clear separation between the embedded region and the surrounding and the treatment of the boundary atoms is not always obvious [26,93]. For systems, in which classical electrostatics dominate such as ionic or molecular solids, the separation between ions and molecules is natural. However, for covalently bonded systems it becomes more difficult to define the QM:MM partitioning. Thus, typically covalent bonds are cut at the QM:MM boundary, which produces dangling bonds that need to be saturated. A multitude of models with different levels of accuracies have been developed to tackle these issues. One example is the CHEMSHELL framework [94,95] that supports Hartree-Fock and hybrid functionals in the embedded region and that has recently been coupled to FHI-AIMS [39].

Another popular approach is "our own $N$-layer integrated molecular orbital molecular mechanics" (ONIOM) by Morokuma and co-workers [25,96]. ONIOM is a so-called extrapolative (or subtractive) scheme in which the total energy of the whole system is given by

$$
E_{\mathrm{ONIOM}}=E_{\mathrm{RL}}-E_{\mathrm{ML}}+E_{\mathrm{MH}},
$$

where the RL refers to the real (or full) system at the lower level, ML refers to the model (or embedded) system at the lower level, and MH labels the model system for the higher level theory. In contrast to the additive QM:MM scheme, ONIOM does not need an additional coupling Hamiltonian to describe the QM/MM interation. When a QM/MM boundary cuts through a covalent bond, link atoms (mostly hydrogen atoms) are added to cap the unsaturated QM boundary for the model calculations. Even if it is common to use MM methods for describing the surroundings, the ONIOM scheme was recently extended to deal with two-layer two-QM embedding, ONIOM(QM1:QM2), where HF was used for the surroundings and the embedded model region is described by MP2 or B3LYP. The QM1/QM2 interactions, including electrostatic interaction, mutual polarization, and charge transfer, are described at the lower QM level.

Our RDMFE scheme is distinctly different from the ONIOM(QM1:QM2) approach. First, the RDMFE scheme is formulated in terms of Green's functions, whereas ONIOM is based on a partition of total energies. As such, spectral properties come out naturally from RDMFE, while the evaluation of total energies is more involved, as discussed in Sec. II E. The

TABLE III. Exchange (X) and correlation (C) components of the $\operatorname{RDMFE}(G W)$ total energy, $E_{\mathrm{XC}}^{G W}\left[G^{\mathrm{emb}}\right]$, as given by Eq. (37) in the limit of an isolated unit cell (large lattice constants): a benchmark against the standard $\operatorname{sc} G W$ calculation for finite systems [49], labeled by $E_{\mathrm{XC}}^{G W}\left[G^{\mathrm{sc} G W}\right]$. All energies are in eV.

\begin{tabular}{lccc}
\hline \hline Term & He & $\mathbf{H}_{2}$ & $\mathbf{N a}_{2}$ \\
\hline$E_{\mathrm{X}}^{G W}\left[G^{\mathrm{emb}}\right]=\frac{1}{2} \int \frac{d \omega}{2 \pi i} \sum_{\mathrm{X}}^{\mathrm{loc}} G^{\mathrm{emb}}(i \omega) e^{-i \omega 0^{+}}$ & -27.173906 & -17.503642 & -760.630196 \\
$E_{\mathrm{X}}^{G W}\left[G^{\mathrm{sc} G W}\right]=\frac{1}{2} \int \frac{d \omega}{2 \pi i} \sum_{\mathrm{X}}^{\mathrm{sc} G W} G^{\mathrm{sc} G W}(i \omega) e^{-i \omega 0^{+}}$ & -27.181325 & -17.505250 & -760.632183 \\
$E_{\mathrm{C}}^{G W}\left[G^{\mathrm{emb}}\right]=\frac{1}{2} \int \frac{d \omega}{2 \pi i} \sum_{\mathrm{C}}^{\mathrm{loc}}(i \omega) G^{\mathrm{emb}}(i \omega) e^{-i \omega 0^{+}}$ & -1.744578 & -2.3053908 & -2.457104 \\
$E_{\mathrm{C}}^{G W}\left[G^{\mathrm{sc} G W}\right]=\frac{1}{2} \int \frac{d \omega}{2 \pi i} \sum_{\mathrm{C}}^{\mathrm{sc} G W}(i \omega) G^{\mathrm{sc} G W}(i \omega) e^{-i \omega 0^{+}}$ & -1.737910 & -2.304200 & -2.455707 \\
\hline \hline
\end{tabular}



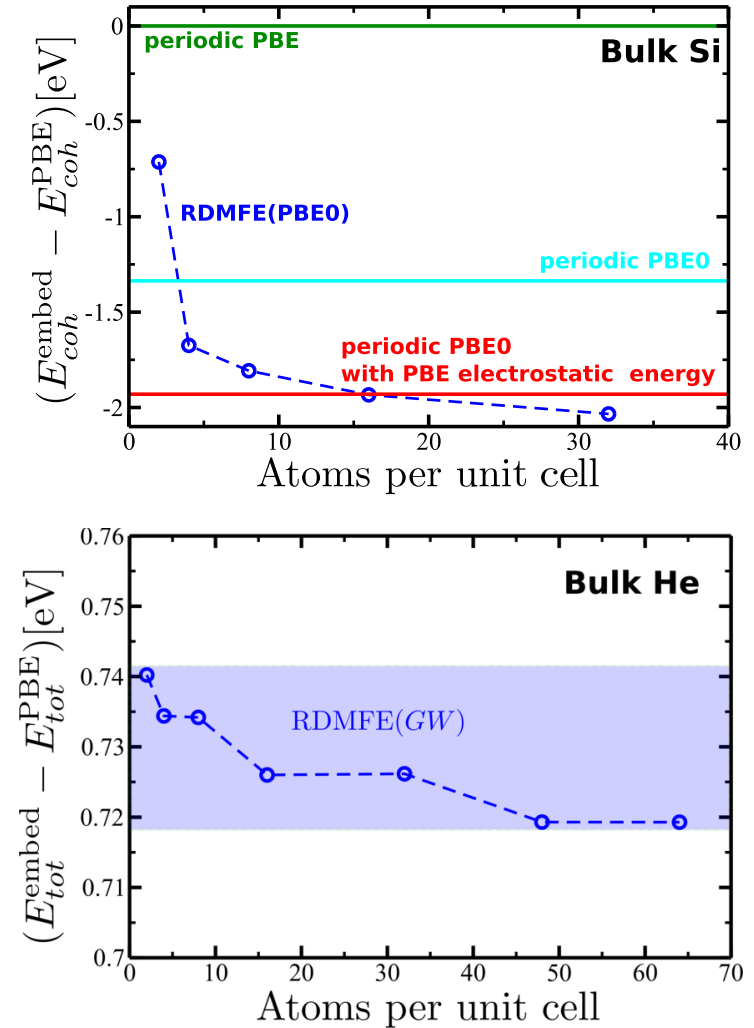

FIG. 12. Upper panel: the RDMFE(PBE0) cohesive energy for bulk Si for increasing unit-cell size. Lower panel: the $\operatorname{RDMFE}(G W)$ total energy for bulk He for increasing unit-cell size, where the blue region indicates a change in the range of $\sim 20 \mathrm{meV}$. Both curves are referenced to the PBE total energy.

opposite is true for the ONIOM(QM1:QM2) scheme. Second, within RDMFE, the effect of the environment is encoded in the bath Green's function that describes an electron reservoir with which the embedded cluster can exchange electrons freely. In other words, the electronic states in the embedded system are not forced to localize within the cluster, but are allowed to delocalize into the surrounding system. Thus, dangling bonds pose no conceptual problem and boundary effects are not significant since they diminish quickly as the size of the cluster increases. In contrast, in ONIOM(QM1:QM2), like in most other embedding schemes in computational chemistry, link atoms are needed to saturate the dangling bonds when chemical bonds are broken. Therefore, ONIOM(QM1:QM2) is most appropriate for describing systems with localized electrons, whereas RDMFE has no problem in dealing with delocalized electrons, especially metallic systems. Third, RDMFE, as is formulated right now, is only applicable to periodic systems that are relevant to solid-state physics, while ONIOM(QM1:QM2) is most suitable for describing molecules and clusters that are of interest to chemical and biological applications.

Addressing the problem of $\mathrm{CO}$ adsorption on $\mathrm{Cu}(111)$ [18], $\mathrm{Hu}$, Reuter, and Scheffler [30,93] developed a cluster extrapolation scheme that is based on performing a cheap (LDA/GGA) calculation for the periodic system then correcting the resulting total energy by $\Delta E_{\mathrm{XC}}=E_{\mathrm{XC}}^{\text {cluster }}[\mathrm{LDA} /$

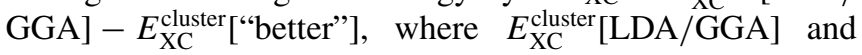
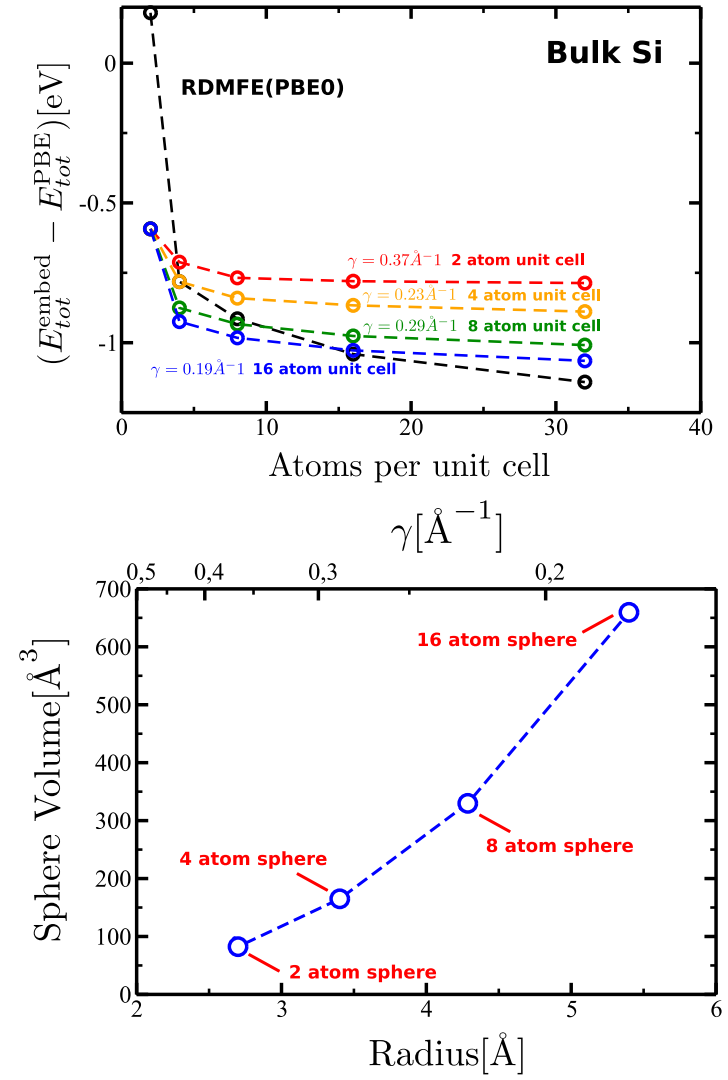

FIG. 13. Upper panel: the RDMFE(HSE) total energy for bulk Si for increasing unit-cell size and different screening parameter $\gamma$. The range-separated total energy converges faster than the PBE0 one (black curve). All the RDMFE(HSE) total energies are shifted to the 2-atom unit-cell value of the embedded PBE0 curve to allow better comparison. Lower panel: the change of the sphere radius and screening parameter $\gamma$ (on a reciprocal scale) with the volume of the sphere surrounding the 2-, 4-, 8-, and 16-atom unit cells.

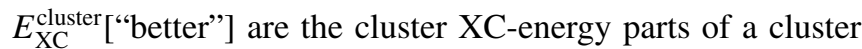
calculation with the cheaper (LDA/GGA) and the "better" theory, respectively, while the cluster itself has been cut out from the periodic system. Increasing the cluster size, they could then show that the correction $\Delta E_{\mathrm{XC}}$ converges for relatively small cluster sizes ( $\sim 16$ atoms) and thus much faster

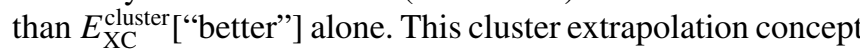
is similar to that of ONIOM(QM1:QM2) described above, but link atoms were not used for the cluster calculations.

Whitten and co-workers also approached molecular adsorbates on metal surfaces [28]. They developed an embedding scheme that builds on identifying a localized subspace that has maximal exchange overlap with the valence orbitals of the atoms within and bordering the adsorbate. The localized subspace is then solved using the CI method, for a fixed Coulomb and exchange potential constructed from the localized orbitals. However, the approach mimics the real periodic system using a large cluster of atoms, which fails in describing the system accurately. Additionally, no systematic cluster extrapolation has been studied. In a similar spirit, Huang and Carter developed a density-functional-based embedding scheme [29]. The scheme relies on the fact that the density is additive, i.e., 

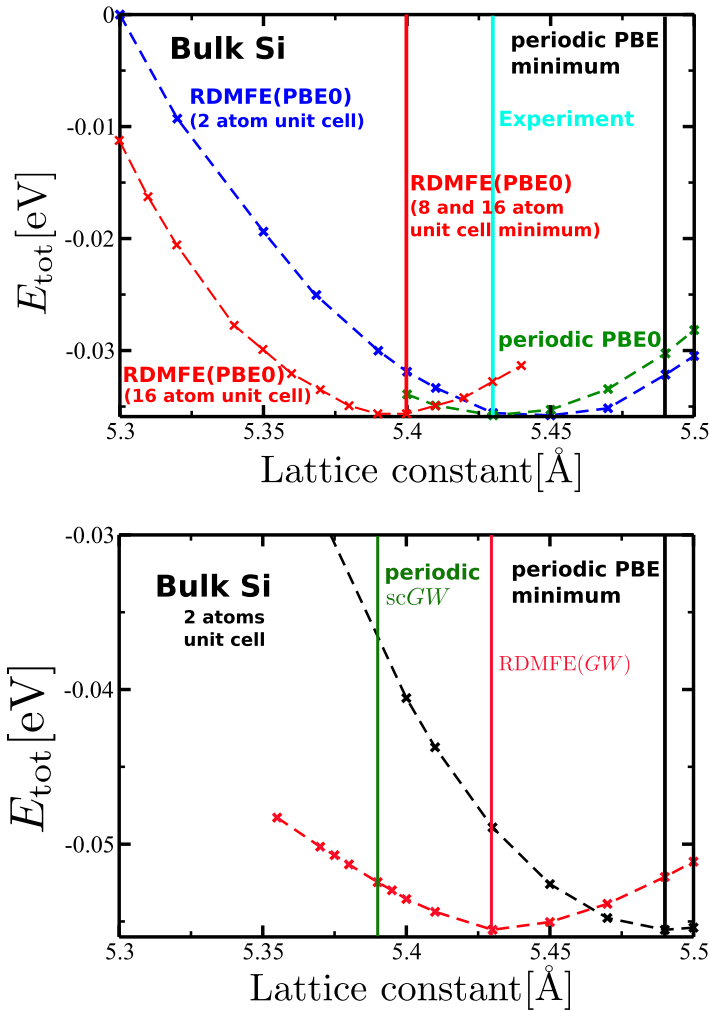

FIG. 14. The RDMFE total energy for bulk $\mathrm{Si}$ as a function of the lattice constant: RDMFE(PBE0) is shown in the upper panel, where the values for the 2-atom unit cell (blue curve) and 16-atom unit cell (red curve) are compared to the periodic PBE0 (green curve) and experiment [88] (vertical cyan solid line). The equilibrium lattice constant for the 8- and 16-atom unit cells is indicated with the vertical red solid line. The $\operatorname{RDMFE}(G W)$ total energy (red curve) is illustrated in the lower panel. Comparison is made with the periodic PBE (black curve). Lattice constant from periodic $\mathrm{sc} G W$ from the work of Kutepov et al. [54] is indicated by the vertical green solid line.

that the total electron density can be partitioned into the density of the embedded region and the density of the embedding surrounding. Proceeding as such allows the definition of an embedding density potential, that is a functional of the total and the embedded density. Adding this potential to the embedded Hamiltonian and solving the resulting $\mathrm{KS}$ Schrödinger equation self-consistently leads to the desired embedded density. For the embedded region, correlated wavefunction methods are typically used, while for the embedding potential the optimized effective potential method or kinetic energy density functionals are employed. Moreover, due to the static nature of the embedding potential, no dynamical methods can be used to describe the embedded region, which limits the applicability to ground-state properties.

For point defects in semiconductors, Scheffler et al. [26] devised a self-consistent Green's function method to compute the change in density induced by the presence of the defect. They considered this change as being a perturbation to the perfect crystal and solved the resulting Dyson equation selfconsistently. Using the fact that defects are well localized in real space, they correct the Hellmann-Feynman force of the perfect crystal, calculated with force fields, by a contribution containing the change in density due to the defect, calculated with KS-DFA. They showed that the resulting HellmannFeynman force is comparable in accuracy to a full DFA calculation.

Finally, it is also worth mentioning that we perform fully self-consistent $G W$ calculations [45,50] in our scheme, which is conceptually different from the so-called quasiparticle self-consistent $G W$ concept [55,56] (QPsc $G W)$. In QPsc $G W$ a series of $G_{0} W_{0}$ calculations is performed. In each iteration, the "best" $G_{0}$ is determined that most closely resembles the $G W$ Green's function of the current cycle. In practice, a static, nonlocal potential is constructed that approximates the $G_{0} W_{0}$ self-energy. This nonlocal potential defines a new noninteraction Hamiltonian $H_{0}$ that produces a new input Green's function $G_{0}$. Since the QPsc $G W$ concept also requires the calculation of the full nonlocal $G_{0} W_{0}$ self-energy, our expectation is that it will be easier to go beyond $G W$ in our RDMFE framework.

\section{CONCLUSIONS}

We have presented an embedding scheme for periodic systems based on Green's functions in the DMFT framework, which maps an infinite periodic system to a single-site (single unit cell) problem coupled to an electronic bath that needs to be determined self-consistently. Our RDMFE Green's function mapping allows a natural definition of the embedded region and defines a self-consistency loop which, at convergence, yields self-consistent Green's functions. The coupling to the surrounding is of dynamical nature enabling electron exchange between the embedded region and the surrounding. We showed that our scheme produces densities of states and total energies that converge well with increasing size of the embedded region. We also demonstrated that the main features of the "better" theory are rapidly captured within our scheme; for example, the plasmon satellite already appears in $\operatorname{RDMFE}(G W)$ calculations for two atoms in the Si unit cell. RDMFE is therefore a promising embedding scheme, that has the potential to make sophisticated and computationally expensive first-principles theories available for periodic systems.

TABLE IV. Bulk Si equilibrium lattice constant and bulk moduli $B_{0}$ for the RDMFE(PBE0) and RDMFE( $\left.G W\right)$. Comparison is made with periodic PBE0 performed with FHI-AIMS, the periodic $\operatorname{sc} G W$ work of Kutepov et al. [54], and experiment [88,90].

\begin{tabular}{lccccccc}
\hline \hline & \multicolumn{3}{c}{ RDMFE(PBE0) } & Periodic PBE0 & RDMFE $(G W)$ & Periodic scGW [54] & Experiment \\
\hline Unit-cell size & 2 atoms & 8 atoms & 16 atoms & 2 atoms & 2 atoms & 5.39 & $5.43[88]$ \\
Lattice constant $(\AA)$ & 5.45 & 5.40 & 5.40 & 5.43 & 5.43 & 100.7 & $99[90]$ \\
Bulk modulus $B_{0}(\mathrm{GPa})$ & 95.14 & 129.07 & 101.33 & 84 & 80.37 & 57 \\
\hline \hline
\end{tabular}




\section{ACKNOWLEDGMENTS}

X.R. acknowledges the helpful discussion with Professor D. Vollhardt and Professor L. Chioncel. This work was supported by the Academy of Finland through its Centres of Excellence
Programme under Projects No. 251748 and No. 284621. Work at University of Science and Technology of China was supported by the Chinese National Science Foundation Grant No. 11374276.
[1] J. P. Perdew and A. Zunger, Phys. Rev. B 23, 5048 (1981).

[2] J. P. Perdew, R. G. Parr, M. Levy, and J. L. Balduz, Phys. Rev. Lett. 49, 1691 (1982).

[3] P. Mori-Snchez, A. J. Cohen, and W. Yang, J. Chem. Phys. 125, 201102 (2006).

[4] J. P. Perdew and M. Levy, Phys. Rev. Lett. 51, 1884 (1983).

[5] L. J. Sham and M. Schlüter, Phys. Rev. Lett. 51, 1888 (1983).

[6] P. Mori-Sánchez, A. J. Cohen, and W. Yang, Phys. Rev. Lett. 100, 146401 (2008).

[7] O. Gunnarsson and B. I. Lundqvist, Phys. Rev. B 13, 4274 (1976).

[8] J. F. Dobson and J. Wang, Phys. Rev. Lett 82, 2123 (1999).

[9] A. Tkatchenko and M. Scheffler, Phys. Rev. Lett. 102, 073005 (2009).

[10] I. D. White, R. W. Godby, M. M. Rieger, and R. J. Needs, Phys. Rev. Lett. 80, 4265 (1998).

[11] K. S. Thygesen and A. Rubio, Phys. Rev. Lett. 102, 046802 (2009).

[12] C. Freysoldt, P. Rinke, and M. Scheffler, Phys. Rev. Lett. 103, 056803 (2009).

[13] T. C. Leung, X. W. Wang, and B. N. Harmon, Phys. Rev. B 37, 384 (1988).

[14] J. Zaanen, O. Jepsen, O. Gunnarsson, A. Paxton, O. Andersen, and A. Svane, Phys. C (Amsterdam) 153-155, 1636 (1988).

[15] W. E. Pickett, Rev. Mod. Phys. 61, 433 (1989).

[16] L. F. Mattheiss, Phys. Rev. B 5, 290 (1972).

[17] L. F. Mattheiss, Phys. Rev. B 5, 306 (1972).

[18] P. J. Feibelman, B. Hammer, J. K. Nørskov, F. Wagner, M. Scheffler, R. Stumpf, R. Watwe, and J. Dumestic, J. Phys. Chem. B 105, 4018 (2001).

[19] I. Mehdaoui and T. Klner, J. Phys. Chem. A 111, 13233 (2007).

[20] I. Mehdaoui and T. Klüner, Phys. Rev. Lett. 98, 037601 (2007).

[21] I. Mehdaoui and T. Klner, Phys. Chem. Chem. Phys. 10, 4559 (2008).

[22] D. Zgid and G. K.-L. Chan, J. Chem. Phys. 134, 094115 (2011).

[23] U. C. Singh and P. A. Kollman, J. Comput. Chem. 7, 718 (1986).

[24] M. J. Field, P. A. Bash, and M. Karplus, J. Comput. Chem. 11, 700 (1990).

[25] F. Maseras and K. Morokuma, J. Comput. Chem. 16, 1170 (1995).

[26] M. Scheffler, J. P. Vigneron, and G. B. Bachelet, Phys. Rev. B 31, 6541 (1985).

[27] J. Bormet, J. Neugebauer, and M. Scheffler, Phys. Rev. B 49, 17242 (1994).

[28] J. L. Whitten and T. A. Pakkanen, Rev. Phys. B 21, 4357 (1980).

[29] C. Huang and E. A. Carter, J. Chem. Phys. 135, 194104 (2011).

[30] Q.-M. Hu, K. Reuter, and M. Scheffler, Phys. Rev. Lett. 98, 176103 (2007).

[31] X. Ren, P. Rinke, and M. Scheffler, Phys. Rev. B 80, 045402 (2009).
[32] W. Metzner and D. Vollhardt, Phys. Rev. Lett. 62, 324 (1989).

[33] A. Georges and G. Kotliar, Rev. Phys. B 45, 6479 (1992).

[34] A. Georges, G. Kotliar, W. Krauth, and M. J. Rozenberg, Rev. Mod. Phys. 68, 13 (1996).

[35] G. Knizia and Garnet Kin-Lic Chan, Phys. Rev. Lett. 109, 186404 (2012).

[36] A. Szabo and N. S. Ostlund, Modern Quantum Chemistry: Introduction to Advanced Electronic Structure Theory (McGraw-Hill, New York, 1989).

[37] J. P. Perdew and K. Schmidt, in Density Functional Theory and its Application to Materials, edited by V. Van Doren, C. Van Alsenoy, and P. Geerlings (AIP, Melville, NY, 2001).

[38] L. Hedin, Phys. Rev. 139, A796 (1965).

[39] D. Berger, A. J. Logsdail, H. Oberhofer, M. R. Farrow, C. R. A. Catlow, P. Sherwood, A. A. Sokol, V. Blum, and K. Reuter, J. Chem. Phys. 141, 024105 (2014).

[40] P. W. Anderson, Rev. Phys. B 124, 41 (1961).

[41] G. Kotliar, S. Savrasov, K. Haule, V. Oudovenko, O. Parcollet, and C. Marianetti, Rev. Mod. Phys. 78, 865 (2006).

[42] K. Held, Adv. Phys. 56, 829 (2007).

[43] V. Blum, F. Hanke, R. Gehrke, P. Havu, V. Havu, X. Ren, K. Reuter, and M. Scheffler, Comput. Phys. Commun. 180, 2175 (2009).

[44] V. Havu, V. Blum, P. Havu, and M. Scheffler, J. Comput. Phys. 228, 8367 (2009).

[45] X. Ren, P. Rinke, V. Blum, J. Wieferink, A. Tkatchenko, A. Sanfilippo, K. Reuter, and M. Scheffler, New J. Phys. 14, 053020 (2012).

[46] S. V. Levchenko, X. Ren, J. Wieferink, R. Johanni, P. Rinke, V. Blum, and M. Scheffler, Comput. Phys. Commun. 192, 60 (2015).

[47] A. Stan, N. E. Dahlen, and R. van Leeuwen, Europhys. Lett. 76, 298 (2006).

[48] C. Rostgaard, K. W. Jacobsen, and K. S. Thygesen, Phys. Rev. B 81, 085103 (2010).

[49] F. Caruso, P. Rinke, X. Ren, M. Scheffler, and A. Rubio, Phys. Rev. B 86, 081102(R) (2012).

[50] F. Caruso, P. Rinke, X. Ren, A. Rubio, and M. Scheffler, Phys. Rev. B 88, 075105 (2013).

[51] F. Caruso, D. R. Rohr, M. Hellgren, X. Ren, P. Rinke, A. Rubio, and M. Scheffler, Phys. Rev. Lett. 110, 146403 (2013).

[52] P. Koval, D. Foerster, and D. Sánchez-Portal, Phys. Rev. B 89, 155417 (2014).

[53] A. Kutepov, S. Y. Savrasov, and G. Kotliar, Phys. Rev. B 80, 041103 (2009).

[54] A. Kutepov, K. Haule, S. Y. Savrasov, and G. Kotliar, Phys. Rev. B 85, 155129 (2012).

[55] M. van Schilfgaarde, T. Kotani, and S. Faleev, Phys. Rev. Lett. 96, 226402 (2006)

[56] T. Kotani, M. van Schilfgaarde, and S. V. Faleev, Phys. Rev. B 76, 165106 (2007). 
[57] P. Werner, A. Comanac, L. de' Medici, M. Troyer, and A. J. Millis, Phys. Rev. Lett. 97, 076405 (2006).

[58] D. Zgid, E. Gull, and Garnet Kin-Lic Chan, Phys. Rev. B 86, 165128 (2012).

[59] S. Y. Savrasov, G. Kotliar, and E. Abrahams, Nature (London) 410, 793 (2001).

[60] K. Held, A. K. McMahan, and R. T. Scalettar, Phys. Rev. Lett. 87, 276404 (2001).

[61] B. Amadon, S. Biermann, A. Georges, and F. Aryasetiawan, Phys. Rev. Lett. 96, 066402 (2006).

[62] J. M. Tomczak, F. Aryasetiawan, and S. Biermann, Phys. Rev. B 78, 115103 (2008).

[63] G. Biroli and G. Kotliar, Phys. Rev. B 65, 155112 (2002).

[64] T. Maier, M. Jarrell, T. Pruschke, and M. H. Hettler, Rev. Mod. Phys. 77, 1027 (2005).

[65] V. I. Anisimov, A. I. Poteryaev, M. A. Korotin, A. O. Anokhin, and G. Kotliar, J. Phys.: Condens. Matter 9, 7359 (1997).

[66] A. I. Lichtenstein and M. I. Katsnelson, Phys. Rev. B 57, 6884 (1998).

[67] S. Biermann, F. Aryasetiawan, and A. Georges, Phys. Rev. Lett. 90, 086402 (2003).

[68] S. Florens, Phys. Rev. Lett. 99, 046402 (2007).

[69] K. Haule, C.-H. Yee, and K. Kim, Rev. Phys. B 81, 195107 (2010).

[70] J. P. Perdew, K. Burke, and M. Ernzerhof, Phys. Rev. Lett 77, 3865 (1996).

[71] A. D. Becke, J. Chem. Phys. 98, 5648 (1993).

[72] J. Heyd, G. E. Scuseria, and M. Ernzerhof, J. Chem. Phys. 118, 8207 (2003).

[73] X. Ren, N. Marom, F. Caruso, M. Scheffler, and P. Rinke, Phys. Rev. B 92, 081104(R) (2015).

[74] M. Ernzerhof and G. E. Scuseria, J. Chem. Phys. 110, 5029 (1999).

[75] C. Adamo and V. Barone, J. Chem. Phys. 110, 6158 (1999).

[76] W. Kohn, Phys. Rev. Lett. 76, 3168 (1996).

[77] V. M. Galitskii and A. B. Migdal, Zh. Eksp. Teor. Fiz. 34, 239 (1958) [Sov. Phys.-JETP 7, 96 (1958)].

[78] H. N. Rojas, R. W. Godby, and R. J. Needs, Phys. Rev. Lett. 74, 1827 (1995).
[79] Ioffedatabase, http://www.ioffe.ru/SVA/NSM/Semicond/Si/ bandstr.html

[80] G. Biroli, O. Parcollet, and G. Kotliar, Phys. Rev. B 69, 205108 (2004).

[81] A. I. Lichtenstein and M. I. Katsnelson, Phys. Rev. B 62, R9283(R) (2000).

[82] Unit cells larger than the primitive unit cell give rise to a folded band structure. To cast the band structure back onto the Brillouin zone of the two 2-atom unit cell we use the unfolding approach of Nemec.

[83] H. Kitamura, J. Phys.: Condens. Matter 25, 065505 (2013).

[84] M. Guzzo, G. Lani, F. Sottile, P. Romaniello, M. Gatti, J. J. Kas, J. J. Rehr, M. G. Silly, F. Sirotti, and L. Reining, Phys. Rev. Lett. 107, 166401 (2011).

[85] J. Lischner, D. Vigil-Fowler, and S. G. Louie, Phys. Rev. Lett. 110, 146801 (2013).

[86] B. Holm and U. von Barth, Phys. Rev. B 57, 2108 (1998).

[87] S. O. Kasapa and P. Capper, in Springer Handbook of Electronic and Photonic Materials (Springer, New York, 2006), Vol. 54, p. 327.

[88] T. Hom, W. Kiszenik, and B. Post, J. Appl. Crystallogr. 8, 457 (1975).

[89] F. Birch, Phys. Rev. 71, 809 (1947).

[90] C. O. Rodríguez, V. A. Kuz, E. L. Peltzer y Blancá, and O. M. Cappannini, Phys. Rev. B 31, 5327 (1985).

[91] M. Gatti, V. Olevano, L. Reining, and I. V. Tokatly, Phys. Rev. Lett. 99, 057401 (2007).

[92] S. Y. Savrasov and G. Kotliar, Phys. Rev. B 69, 245101 (2004).

[93] Q.-M. Hu, K. Reuter, and M. Scheffler, Phys. Rev. Lett. 99, 169903(E) (2007).

[94] P. Sherwood, A. H. de Vries, M. F. Guest, G. Schreckenbach, C. A. Catlow, S. A. French, A. A. Sokol, S. T. Bromley, W. Thiel, A. J. Turner, S. Billeter, F. Terstegen, S. Thiel, J. Kendrick, S. C. Rogers, J. Casci, M. Watson, F. King, E. Karlsen, M. Sjøvoll, A. Fahmi, A. Schäfer, and C. Lennartz, J. Mol. Struct.: (THEOCHEM) 632, 1 (2003).

[95] A. A. Sokol, S. T. Bromley, S. A. French, C. R. A. Catlow, and P. Sherwood, Int. J. Quantum Chem. 99, 695 (2004).

[96] L. W. Chung, H. Hirao, X. Li, and K. Morokuma, WIREs Comput. Mol. Sci. 2, 327 (2012). 